\title{
Threshold harvesting as a conservation or exploitation strategy in population management
}

\author{
Frank M. Hilker ${ }^{1}$. Eduardo Liz $^{2}$ \\ Received: 8 May 2019 / Accepted: 18 May 2020 / Published online: 20 June 2020 \\ (C) The Author(s) 2020
}

\begin{abstract}
Threshold harvesting removes the surplus of a population above a set threshold and takes no harvest below the threshold. This harvesting strategy is known to prevent overexploitation while obtaining higher yields than other harvesting strategies. However, the harvest taken can vary over time, including seasons of no harvest at all. While this is undesirable in fisheries or other exploitation activities, it can be an attractive feature of management strategies where removal interventions are costly and desirable only occasionally. In the presence of population fluctuations, the issue of variable harvests and population sizes becomes even more notorious. Here, we investigate the impact of threshold harvesting on the dynamics of both population size and harvests, especially in the presence of population cycles. We take into account semelparous and iteroparous life cycles, Allee effects, observation uncertainty, and demographic as well as environmental stochasticity, using generic mathematical models in discrete time. Our results show that threshold harvesting enhances multiple forms of population stability, namely persistence, constancy, resilience, and dynamic stability. We discuss plausible choices of threshold values, depending on whether the aim is resource exploitation, pest control, or the stabilization of fluctuations.
\end{abstract}

Keywords Fixed escapement · Harvest control rule $\cdot$ Pest control · Maximum sustainable yield · Intervention frequency · Population cycles $\cdot$ Stability

\section{Introduction}

The management of ecosystems often requires population harvesting. Examples include the control of invasive species, removal of weeds, roguing of infected plants, regulation of pests such as defoliating insects, and the harvesting of fisheries and forestry. The strategy of threshold harvesting removes the entire excess of a population stock above a certain threshold value and takes no harvest if the stock is below the threshold. In many instances, this strategy emerges naturally, e.g., when pests exceed economic injury

Frank M. Hilker

frank.hilker@uni-osnabrueck.de

Eduardo Liz

eliz@dma.uvigo.es

1 Institute of Mathematics and Institute of Environmental Systems Research, Osnabrück University, 49069 Osnabrück, Germany

2 Departamento de Matemática Aplicada II, Universidade de Vigo, 36310 Vigo, Spain levels, detection thresholds, or values that can be used to justify spending a budget. ${ }^{1}$

The threshold is sometimes also called the escapement (or biomass reference point in the fisheries literature), because it corresponds to the population level that "escapes" harvesting. As a harvesting strategy that keeps the population at a constant ceiling, threshold harvesting can be seen as the antipode to the constant-quota harvesting strategy, which removes a constant quota from the population. Proportional harvesting, which removes a constant fraction of the population, corresponds to an intermediate strategy between the two extremes of threshold harvesting and constant-quota harvesting. Figure 1 illustrates the three harvesting strategies by showing their relative harvest mortality

\footnotetext{
${ }^{1}$ Equivalent effects on a population can be caused by mechanisms other than harvesting. The earliest examples we are aware of are given by Ricker (1952) (and reiterated in his famous paper Ricker 1954, Fig. 34 and pp. 609-616). He describes the situation when a population is regulated by generalist predators in such a way that only individuals in refuges of a given size escape predation or that predators switch their search image abruptly at a certain prey threshold density. He refers to two empirical examples, one being predation upon bob-white in Iowa and the other one being predation upon Atlantic salmon parr in some rivers.
} 


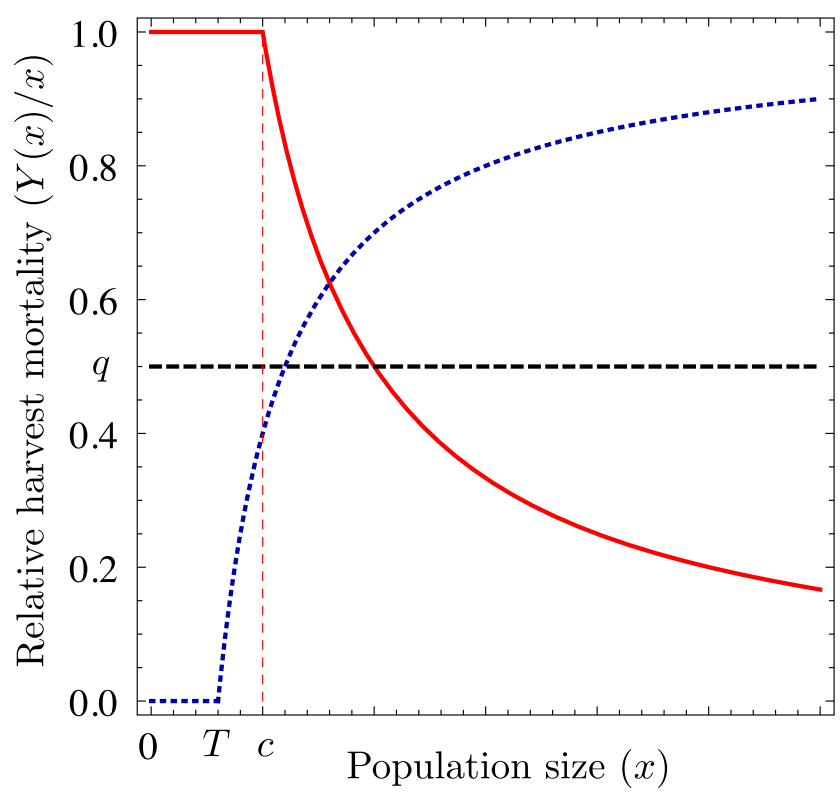

Fig. 1 Relative harvest mortalities for threshold harvesting (blue, dotted), proportional harvesting (black, dashed), and constant-quota harvesting (red, solid). We denote by $x$ the population size at equilibrium, $Y(x)$ the corresponding yield, $T$ the threshold, $c$ the constant quota, and $q$ the proportional rate of harvest

(or per-capita harvest rate) as a function of population abundance. For threshold harvesting, the relative population removal ceases as the population becomes smaller and stops altogether when the population drops below the threshold. By contrast, for constant-quota harvesting, the relative population removal increases as the population becomes smaller. For proportional harvesting, the relative population removal is constant.
Threshold harvesting is known under many different names in quite different disciplines (see Table 1). Here, we use the term "threshold harvesting" because it seems the most inclusive one for the wide range of applications that we have in mind, stretching from pest control to resource exploitation. To avoid confusion, we point out that the term "threshold harvesting" is sometimes also used for other harvesting strategies, where there are different forms of removal above the threshold (e.g., Kaitala et al. 2003; Enberg 2005).

According to a review of harvesting strategies, threshold harvesting generally performs best for maximizing cumulative yield, mean annual yield, and profit in the absence of observation error (Deroba and Bence 2008). This has been found to hold under a wide range of assumptions (e.g., Ricker 1958; Clark 1976; Reed 1978, 1979, 1980; Ludwig 1979, 1980, 1998; Mendelssohn 1979; Mangel 1985; Lande et al. 1995, 1997). As harvesting is curtailed when the population is below the threshold level, threshold harvesting has a built-in mechanism to accommodate random variation in environmental conditions or vital rates (Lande et al. 1997). This has two major consequences. On the one hand, this makes threshold harvesting a precautionary strategy that prevents overexploitation and maintains spawning biomass. On the other hand, threshold harvesting may impose a "stopand-go fishery" (Evans 1981), which implies an economic and political cost to more precautionary harvesting. Ricker (1958) was perhaps the first to observe that the variability of catch is greater for threshold harvesting than for some other strategies (see also Larkin and Ricker 1964; Tautz et al. 1969; Allen 1973; Gatto and Rinaldi 1976). Another drawback of threshold harvesting is that it requires up-todate knowledge of stock size (Hilborn and Walters 1992).
Table 1 A collection of the many names of threshold harvesting

\begin{tabular}{ll}
\hline Name & References \\
\hline $\begin{array}{l}\text { Ecology, fisheries, natural resources management } \\
\text { Threshold harvesting }\end{array}$ & \\
Fixed escapement & Lande et al. (1995) and Lande et al. (1997) \\
Constant escapement & Hilborn (1979) and Fryxell et al. (2005) \\
Fixed stock & Reed (1979) and Getz and Haight (1989) \\
Constant-stock-size strategy & Jonzén et al. (2002) \\
Optimal strategy & Hilborn and Walters (1992) \\
Bang-bang & Ludwig (1998) \\
Optimum stabilization of escapement & Clark (1976) \\
Trigger harvest & Ricker (1958) \\
(upper) limiter control & Sabo (2005) \\
Physics, telecommunication, nonlinear dynamics & Hilker and Westerhoff (2006) and Tung et al. (2014) \\
Thresholding & Sinha (1994) and Sinha (2001) \\
Flat-topped maps & Glass and Zeng (1994) \\
Simple limiter control & Corron et al. (2000) and Stoop and Wagner (2003) \\
\hline
\end{tabular}


Errors in stock size estimates may (Engen et al. 1997; Milner-Gulland et al. 2001; Lillegård et al. 2005) or may not (Butterworth and Bergh 1993; Polacheck et al. 1999) change the relative superiority of threshold harvesting compared to other strategies. In practice, thresholds are typically set too low (Ludwig et al. 1993). Yet, it remains that threshold harvesting is a strategy minimizing extinction risk while optimizing yield (Lande et al. 1995; Lande et al. 1997) and could thus bring together conservation and exploitation interests.

Recent microcosm experiments have investigated threshold harvesting of populations of the ciliate Tetrahymena thermophila (Fryxell et al. 2005) and the fruit fly Drosophila melanogaster (Tung et al. 2016), for selected threshold values. Both studies support the utility of threshold harvesting as a management strategy. In both experiments, threshold harvesting was found to reduce variability in population density and to reduce extinction risk.

Depending on the context, harvesting may be desirable because it generates revenue (e.g., in fisheries) or undesirable because it causes expenditures (e.g., in pest control). In this paper, we focus on three areas of application, namely exploitation, pest control, and the dynamic stabilization of fluctuating populations. Populations with fluctuations present unique challenges to managers (Barraquand et al. 2017); for instance, the harvest can vary dramatically between years and sometimes stop completely. While the latter is desirable for pest control, it can be hazardous for fishing industries. We will consider population fluctuations that are endogenously generated (due to overcompensation, time lags, or nonlinear population interactions), including the case where there are additionally exogenous fluctuations (due to random variations in the biology and in the physical environment). This differs from previous work by Lande and colleagues (reviewed in Lande et al. 1997), which considers stochastic fluctuations in dynamically stable populations and assumes the absence of endogenously generated oscillations.

Harvesting is perceived as magnifying exogenous and reducing endogenous fluctuations (Milner-Gulland and Mace 1998). For a more informed basis, we consider different forms of stability, namely persistence stability (persisting through time as opposed to going extinct); constancy stability (population size remaining essentially unchanged); dynamic stability (eventually returning to the equilibrium after perturbations); and resilience (the ability to absorb perturbations that are within the domain of attraction) (Grimm and Wissel 1997). In the next Section, we investigate the effect of threshold harvesting on these forms of stability for semelparous and iteroparous species. "Models with Allee effect" considers populations with demographic Allee effect. "Average long-term yield" focuses on the average long-term yield. "Stochastic simulations" takes into account stochasticity in the form of demographic and autocorrelated environmental variations as well as observation error, and investigates the harvest frequency and the variability of yield as a function of harvesting intensity. When we say increased threshold harvesting we mean setting a lower threshold (escapement) which amounts to elevated harvesting intensity. In each of these sections, we not only present results, but also place the models in the context of the literature and discuss the relevance of our findings. "Conclusions" provides a summary of the results and draws overall conclusions.

\section{Population dynamic effects of threshold harvesting}

In this Section, we study single-species populations without Allee effect managed by threshold harvesting. Let $x_{n}$ be the population size at time step $n, n=0,1,2, \ldots$. The population dynamics in the absence of harvesting is assumed to follow

$x_{n+1}=f\left(x_{n}\right)$

for an initial value $x_{0}>0$. The map $f$ gives the discrete-time reproduction. In this Section, we assume a typical condition for $f$, which is satisfied by semel- and iteroparous population models without Allee effect, such as the Beverton-Holt, Ricker, Hassell or Ricker-Clark maps:

(A) $f:[0, \infty) \rightarrow[0, \infty)$ is continuous, has a unique positive fixed point $K, f(x)>x$ for $x \in(0, K)$, and $0<f(x)<x$ for $x>K$.

If the population is managed by threshold harvesting, then the dynamics is governed by the difference equation

$x_{n+1}=\min \left\{f\left(x_{n}\right), T\right\}:=g\left(x_{n}\right)=\left\{\begin{array}{cl}f\left(x_{n}\right) & \text { if } f\left(x_{n}\right) \leq T, \\ T & \text { if } f\left(x_{n}\right)>T,\end{array}\right.$

where $T>0$ is the threshold. The smaller the value of $T$, the larger the harvesting intervention. The graph of Eq. (2.2) is a truncated map as illustrated in Fig. 2a, c.

Next, we will state a few properties regarding the dynamic stability of a managed population, depending on whether the population is stable or unstable in the absence of harvesting.

\section{Dynamic stability}

Here, we will show that threshold harvesting can never have a destabilizing effect on the managed population. By destabilizing, we mean that an equilibrium that is locally asymptotically stable in the absence of harvesting becomes unstable due to harvesting. Instead, we will show 
Fig. 2 Examples of threshold harvesting with $T=50$ (top row) and $T=80$ (bottom row). In both cases, the unmanaged population cycles with period 4, following a Ricker map with $r=2.6$ and $K=60$. Diagrams in the left column show the unmanaged Ricker map (humpshaped) and the harvested population maps (flat-topped). Threshold harvesting begins after ten time steps. For the low threshold (top row), harvesting stabilizes the population on a stable equilibrium (b), shown as red point in (a). For the large threshold (bottom row), harvesting changes the period of the population cycle from 4 to 2 (d), shown in thin dotted line in (c)
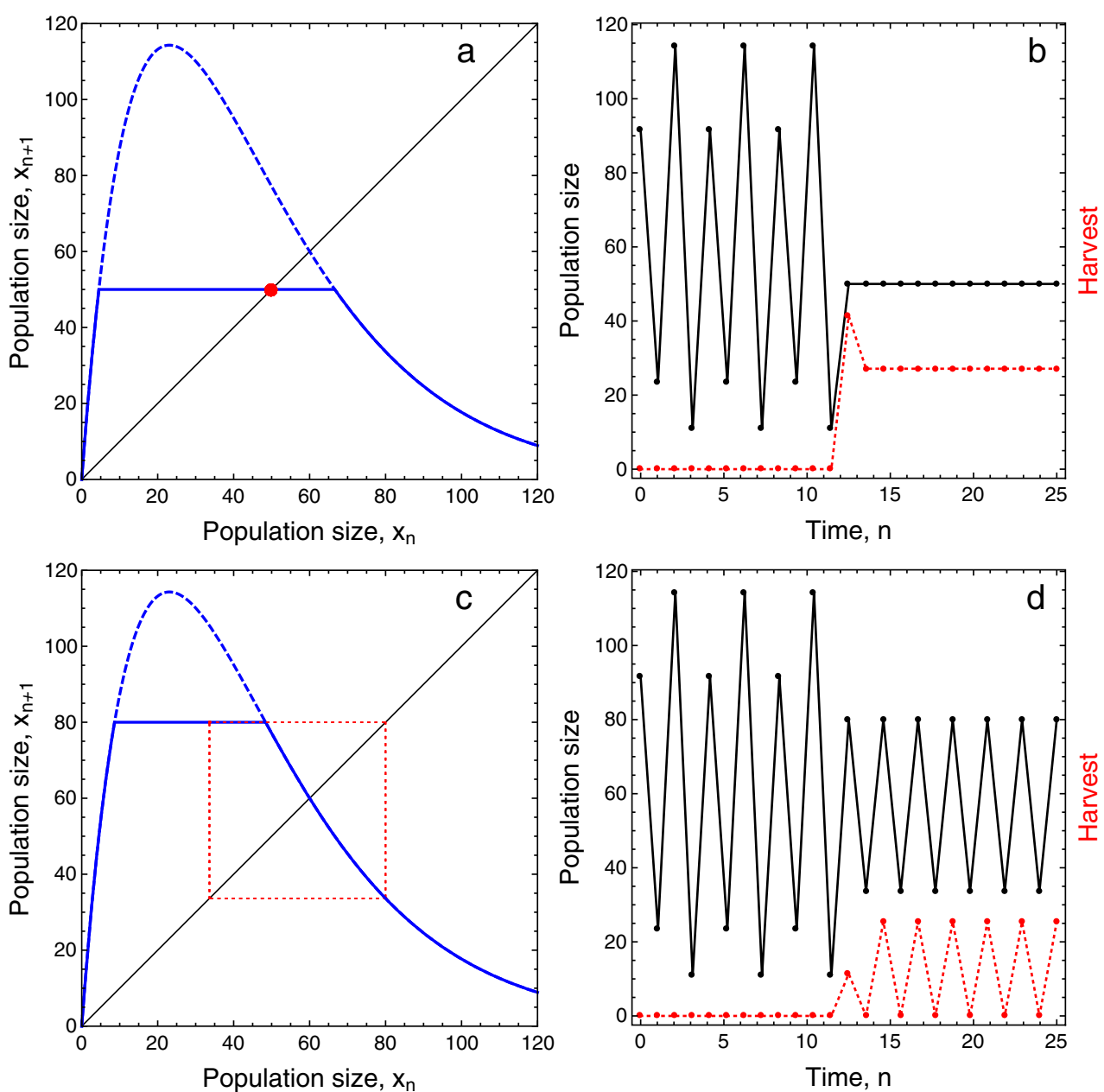

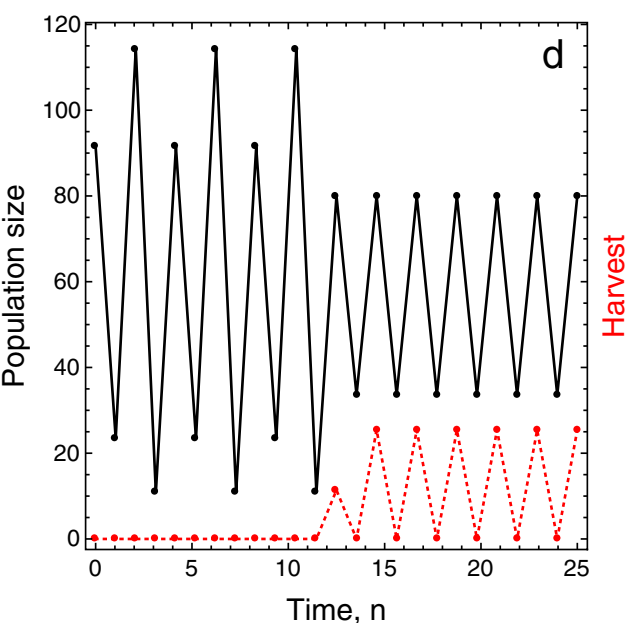

that strong enough harvesting, i.e., a small escapement, stabilizes population dynamics.

In fact, if the threshold is set below the carrying capacity $(T<K)$, the system has a stable equilibrium. See Fig. 2a, b for an example. That is, for threshold values $T<K$, the map $g$ has a unique positive equilibrium $K=T$, and all solutions of Eq. (2.2) starting at an initial condition $x_{0}>0$ converge to $K$. If this property holds, we say that $K$ is a global attractor. We state this property in the following proposition, whose proof can be found in Appendix A.1:

Proposition 2.1 Assume that $f$ satisfies $(\boldsymbol{A})$. If $T \leq K$, then $T$ is the unique positive equilibrium of $\mathrm{Eq}$. (2.2), and it is a global attractor.

In the remainder of this section, we consider the scenario that the threshold is set at a value greater than the carrying capacity $(T>K)$. Then, the dynamics of the managed system (2.2) depends on the dynamics of the unmanaged system (2.1). We distinguish three cases, namely where the unmanaged population (i) has a stable equilibrium, (ii) is periodic, or (iii) chaotic.
First, consider the dynamically simplest case. So we assume that the positive equilibrium $K$ is a global attractor of Eq. 2.1. The following result states that this equilibrium cannot be destabilized by threshold harvesting:

Proposition 2.2 Assume that $f$ satisfies (A), and $K$ is a global attractor of Eq. (2.1). If $T \geq K$, then $K$ is the unique positive equilibrium of Eq. (2.2), and it is a global attractor.

This result is a particular case of a more general result proved in Hilker and Liz (2019, Proposition A.3).

Second, consider that the equilibrium $K$ of Eq. (2.1) is unstable and that Eq. (2.1) has a finite number of periodic orbits. We will show that increased harvesting pressure, i.e., decreasing the threshold, induces a sequence of period-halving bifurcations until the equilibrium becomes stable. It is possible to determine the threshold value for desired periods, which includes the threshold necessary for stabilization. Figure $2 \mathrm{c}$ and $\mathrm{d}$ show an example where threshold harvesting changes a 4-cycle into a 2-cycle.

An application of Sharkovsky's theorem on the coexistence of cycles (see Sharkovsky et al. 1997) ensures that 
all the periods are powers of two. For the unimodal reproduction maps usually employed in population dynamics, if $p=2^{N}$ is the largest period of a periodic orbit of $f$, then this $p$-periodic orbit is unique, and it attracts all initial conditions except those belonging to the other periodic orbits and their preimage. Denote the attracting periodic orbit by $O=\left\{x_{1}, x_{2}, \ldots, x_{p}\right\}$, where $x_{1}<\cdots<x_{p}$. As long as $T \geq x_{p}$, it is clear that $O$ attracts almost all initial conditions of Eq. (2.2). As $T$ is decreased below $x_{p}$, a sequence of period-halving bifurcations occurs, in such a way that, for each $T \in\left(K, x_{p}\right)$, system (2.2) has a periodic attractor whose period is $q=2^{m}$, with $1 \leq m \leq N$. This attractor has the form

$\Gamma_{q}=\left\{T, f(T), \ldots, f^{q-1}(T)\right\}$,

where $f^{k}$ denotes, as usual, $k$ iterations of $f$ for any integer $k \geq 1$, that is,

$f^{k}(x)=\underbrace{(f \circ \cdots \circ f)}_{k}(x)$.

The period $q$ in Eq. 2.3 is determined as the least integer such that $f^{q}(T) \geq T$. For example, under assumption (A), the condition $T>K$ is equivalent to $f(T)>T$. If $f(T)<T \leq f^{2}(T)$, then all positive solutions converge to the 2-periodic orbit $\{T, f(T)\}$, with the only exception of $K$ and its preimage (see Fig. 2c).

Figure 3 shows a bifurcation diagram and illustrates the bifurcation conditions for the period-halvings from 4cycles over 2-cycles to stable fixed points when increasing threshold harvesting, i.e., reducing the value of $T$ as the bifurcation parameter. We provide more details in Appendix A.2.

Third and last, consider that the dynamics of the unmanaged map (2.1) is chaotic. As in the previous case, for SU-maps (see Appendix A.2), there is a unique attracting periodic orbit of the managed system (2.2), which has the form of Eq. (2.3). With probability one, all other orbits fall into this stable periodic attractor (see Thunberg 2001, Corollary 5 and Theorem 6). The period $q$ is again determined as the least positive integer for which $f^{q}(T) \geq T$. Since the map $g$ defined in Eq. (2.1) is continuous, there is $\mathrm{Li}-$ Yorke chaos as long as the period of the attracting cycle is not a power of two. However, since there is a periodic attractor, the chaotic repellor is not observable; in particular, the map $g$ does not have sensitive dependence (Thunberg 2001 , p. 15). Once the chaotic dynamics becomes simpler due to threshold harvesting (with periodic attractors having a period $q=2^{m}$ ), we have the situation considered in the previous case. Earlier results in this direction have been reported by Sinha (1994) who studied some properties of thresholding for the chaotic quadratic map $f(x)=1-$ $2 x^{2}$. The bifurcation diagram of Eq. (2.2), using $T$ as the bifurcation parameter, exhibits some characteristic features,

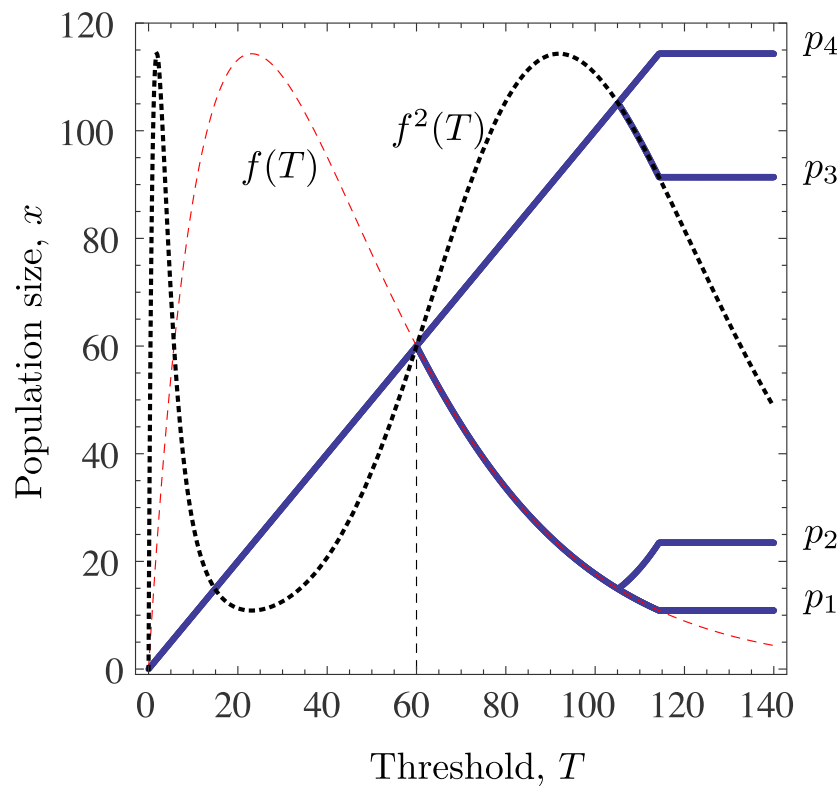

Fig. 3 Bifurcation diagram of the threshold harvesting strategy (2.2), shown in solid blue curve, for the Ricker map $f(x)=x e^{r(1-x / K)}$ with $r=2.6$ and $K=60$. For convenience, we also show the graphs of $f$ (dashed, red) and $f^{2}$ (dotted, black). The bifurcation parameter is the threshold value $T$, ranging between 0 and 140 (a little bit beyond the maximum of $f$ ). The uncontrolled map $f$ has an attracting cycle of period $4=2^{2}$, namely, $\left\{p_{1}, p_{2}, p_{3}, p_{4}\right\}$. For $T \geq p_{4}$, this cycle is also attracting for Eq. 2.2. As long as $T<p_{4}$ and $f^{2}(T)<T$, the attracting cycle is $\left\{T, f(T), f^{2}(T), f^{3}(T)\right\}$. The first period-halving bifurcation occurs at $T=T_{1} \approx 105$, determined by the unique point in $\left(p_{3}, p_{4}\right)$ such that $f^{2}\left(T_{1}\right)=T_{1}$. Another period-halving bifurcation occurs at $T=K=60$, the positive fixed point of $f$. For $T \leq K$, the threshold $T$ is globally attracting (Proposition 2.1)

which include periodic windows and ranges of effectively chaotic behavior; this means that even if there is a periodic attractor, its period can be very large. Further properties of bifurcation diagrams for threshold harvesting when the map $f$ is chaotic can be found in Glass and Zeng (1994), Stoop and Wagner (2003), Zhou and Yu (2005), and Zhou (2006). See also Hilker and Westerhoff $(2005,2006)$ and Hilker and Liz (2013).

\section{Bimodal maps with iteroparity}

A consequence of the dynamic behavior described in "Dynamic stability" is that, for a population stabilized on a periodic cycle, inherent fluctuations cannot be enhanced by threshold harvesting. This is a key difference to other harvesting strategies. For instance, constant-quota harvesting of a population modeled by a Ricker map with a 4-periodic attractor can induce potentially undesirable effects such as increased complexity (e.g., towards chaos), essential extinction, and sudden collapses (Sinha and Parthasarathy 1996; Schreiber 2001). Here, we illustrate that threshold harvesting cannot be destabilizing by focusing on iteroparous 
(or perennial) species that can have multiple reproductive cycles over their lifetime. Iteroparous species are described by bimodal maps, for which both constant-quota and proportional harvesting can be destabilizing (see below).

Although proportional harvesting tends to simplify the dynamics for a unimodal Ricker-type model, it has been recently shown that this is not always the case for the Ricker-Clark model (Clark 1976; Thieme 2003; Liz 2010)

$f(x)=\alpha x+(1-\alpha) x e^{r(1-x / K)}$,

where the survival parameter $\alpha$ describes survivorship of adults from one season to the next. Equation 2.4 has been recently employed by Shelton and Mangel (2011) to study how fishing can magnify fluctuations in fish populations, and by Yakubu et al. (2011) to asses the performance of proportional harvesting. Even if the survival rate is very small, a 2-periodic attractor of Eq. (2.4) can be transformed into a chaotic attractor by proportional harvesting (see Liz and Ruiz-Herrera 2012, Fig. 8). For larger values of $\alpha$, a globally attracting equilibrium can be destabilized by harvesting. For proportional harvesting, this phenomenon has been noticed by Yakubu et al. (2011) and analyzed in detail by Liz and Ruiz-Herrera (2012); for constant-quota harvesting, this effect has been shown in Liz (2010). In view of Propositions 2.1 and 2.2, threshold harvesting cannot destabilize a global attractor. For comparison, we consider Eq. 2.4 with $K=60, r=4$, and $\alpha=0.56$. In this case, the equilibrium $K$ is a global attractor. A strategy of proportional harvesting destabilizes the equilibrium by a period-doubling bifurcation (Fig. 4a). Constant-quota harvesting not only destabilizes the equilibrium, but also induces a strong Allee effect capable of producing essential extinction or a sudden collapse if the catch quota is large enough (Fig. 4b). Finally, in accordance with our analytical results, threshold harvesting does not destabilize the positive equilibrium (Fig. 4c). The corresponding models are

$x_{n+1}=(1-\gamma / M) f\left(x_{n}\right) \quad$ (proportional harvesting),

$x_{n+1}=\max \left\{f\left(x_{n}\right)-d, 0\right\} \quad$ (constant-quota harvesting),

$x_{n+1}=\min \left\{f\left(x_{n}\right), T\right\} \quad$ (threshold harvesting),

where $M \approx 141$ is the maximum of $f$.

\section{Constancy and persistence stability}

One measure of constancy stability is the fluctuation range, and one measure of persistence stability is the inverse of the minimum population size (as extinction risk is larger the smaller the population size). On the basis of these measures, we find that increased threshold harvesting can enhance, but that it never deteriorates constancy stability. Moreover, we find that increased threshold harvesting deteriorates persistence stability for $T<K$ and, somewhat counterintuitively, enhances persistence stability for $T>K$.

As we have mentioned, typical unimodal population maps (e.g., Ricker, Hassell, Maynard-Smith, quadratic) have a unique positive equilibrium $K$ and are decreasing for $x>K$. In this case, if the equilibrium is unstable, a common feature of the bifurcation diagrams in the presence of threshold harvesting (2.2) is that, once the harvesting is implemented, the enveloping curves of the diagram are defined by the line $x=T$ and the curve $x=f(T)$ until the equation has a stable fixed point at $T=K$ (for example, see Fig. 3). The reason is that, since the periodic attractor $\Gamma$ has the form (2.3), it is clear that the maximum value of $\Gamma$ is $T$ and the minimum is $f(T)$. This has two immediate implications. First, the extent of the periodic attractor (i.e., the range of fluctuations) is an increasing function of $T$ $\left(T>K\right.$ implies that $f^{\prime}(T)<0$ and hence $T-f(T)$ is increasing); in other words, constancy stability increases as a

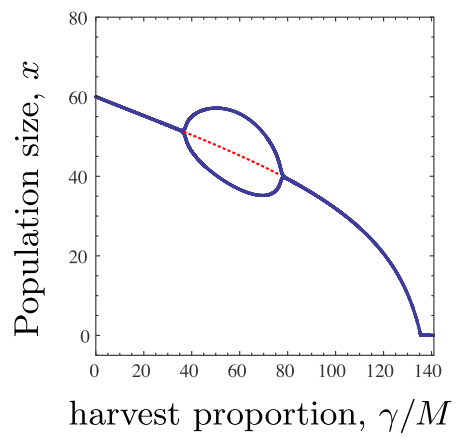

b

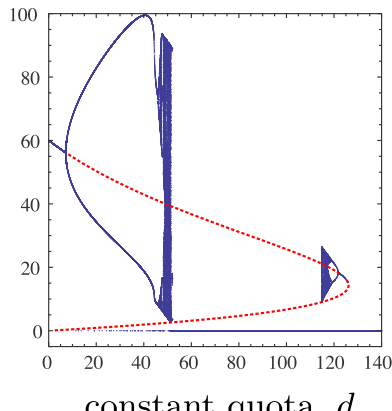

C

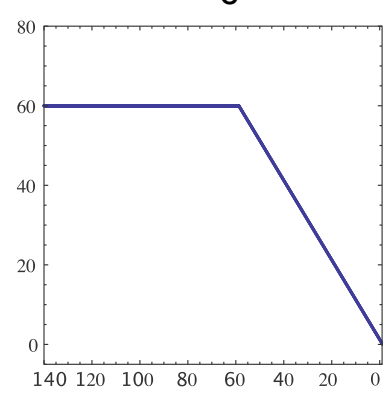

Threshold, $T$
Fig. 4 Bifurcation diagrams for the bimodal Ricker-Clark map (2.4) managed by three different harvesting strategies. a For proportional harvesting, a bubble is created (cf. Liz and Ruiz-Herrera 2012). b For constant-quota harvesting, the equilibrium is destabilized too; note that an additional unstable equilibrium appears due to the induced strong Allee effect, and that there are boundary crises (Schreiber 2001). c Threshold harvesting does not destabilize the equilibrium. Red dashed lines indicate unstable equilibria. Initial conditions drawn from a pseudo-random uniform distribution. Parameter values: $K=$ $60, r=4, \alpha=0.56$. $M \approx 141$ is the maximum value of $f$ 
the intensity of threshold harvesting increases. This property is no longer true for bimodal maps if the cycle has a period larger than two. Second, the minimum population size of the periodic attractor, i.e. $f(T)$, increases with lower values of $T$ as long as $T>K$. As stochastic extinction is more likely for small population sizes, increased threshold harvesting enhances persistence stability for $T>K$. If $T \leq K$ and the population is dynamically stabilized to equilibrium, this is no longer the case.

\section{Models with Allee effect}

In this section, we investigate the effect of threshold harvesting on population dynamics with a strong Allee effect (also called critical depensation in the fisheries literature) in the absence of harvesting. So far, Allee effects are acknowledged to increase extinction risk and to reduce yield as well as yield predictability under threshold harvesting (Ricker 1958; Collie and Spencer 1993; Eggers 1993; Lande et al. 1995; Walters and Parma 1996; Ludwig 1998). Here, we will show that threshold harvesting is a remarkably resilient and adaptive strategy in comparison to constant-quota and proportional harvesting and that it can prevent essential extinction, in which almost all initial conditions would lead to extinction in the absence of harvesting.

We consider the Ricker-Schreiber model

$$
x_{n+1}=\frac{s x_{n}}{1+s x_{n}} x_{n} e^{r\left(1-x_{n} / K\right)}:=f\left(x_{n}\right), \quad n=0,1,2, \ldots,
$$

where parameter $s>0$ represents an individual's efficiency to find a mate (Schreiber 2003, Sect. 2.1). This model has two positive equilibria $K_{1}<K_{2}$, where $K_{2}$ is the carrying capacity and $K_{1}$ is the so-called Allee threshold. There are three generic possibilities for the dynamics of model (3.1): extinction, bistability between extinction and survival, and essential extinction (Schreiber 2001). The latter means that extinction occurs for a randomly chosen initial condition with probability one and results from a combination of overcompensatory instability and the strong Allee effect. Thus, it is natural that any harvesting method can prevent essential extinction when it reduces the undesirable effects of overcompensation. The transition from essential extinction to the existence of a nontrivial attractor takes place via a boundary collision. Some examples for constant-quota harvesting and proportional harvesting can be found in Sinha and Parthasarathy (1996), Schreiber (2001), Liz (2010), and Cid et al. (2014).

Threshold harvesting prevents essential extinction, too. Moreover, the escapement level necessary to avoid essential extinction is very easy to compute: just take the value of $T>K_{2}$ such that $f(T)=K_{1}$, that is, the preimage of $K_{1}$ by the map $f$. If the escapement is set too low, then the species is doomed to extinction through a sudden collapse induced by the Allee effect; however, compared with constant-quota or proportional harvesting, this effect is less dramatic for threshold harvesting since this is only possible if the escapement is below the Allee threshold $K_{1}$. We provide some examples to illustrate these remarks.

First, consider Eq. (3.1) with $K=60$ and $r=5$. Figure 5a shows the stability diagram for $T \in(0,200)$ and $s \in(0.01,0.04)$. The border between essential extinction and bistability is defined by the implicit curve $f(T)=K_{1}$ $\left(T>K_{2}\right)$; the equilibrium $K_{2}$ is attracting when $K_{1}<$ $T \leq K_{2}$; and there is extinction for $T \leq K_{1}$. These generic dynamics can be observed in Fig. $5 b$ for the particular value $s=0.025$ (corresponding to the dashed line in Fig. 5a). Essential extinction can be prevented if $T<T_{1}=131.72$, and there is a nontrivial attractor if $T_{2}<T<T_{1}$, where $T_{2}=K_{1} \approx 0.27$ is the Allee threshold.

Next, we consider Eq. (3.1) with $K=1, r=4$, and $s=0.6$, which has a 2-periodic attractor coexisting with the trivial attractor. We have normalized the carrying capacity to 1 in order to make the figures clearer. In Fig. 6, we observe the effects of harvesting for constantquota, proportional, and threshold harvesting. We focus on sudden collapses due to the interaction between harvesting
Fig. 5 a Stability diagram of the Allee effect model (3.1) managed by threshold harvesting in the parameter plane $(T, s)$ with $K=60$ and $r=5$. b Bifurcation diagram for fixed $s=0.025$ (corresponding to the horizontal dashed line in a). Initial conditions drawn from a pseudo-random uniform distribution
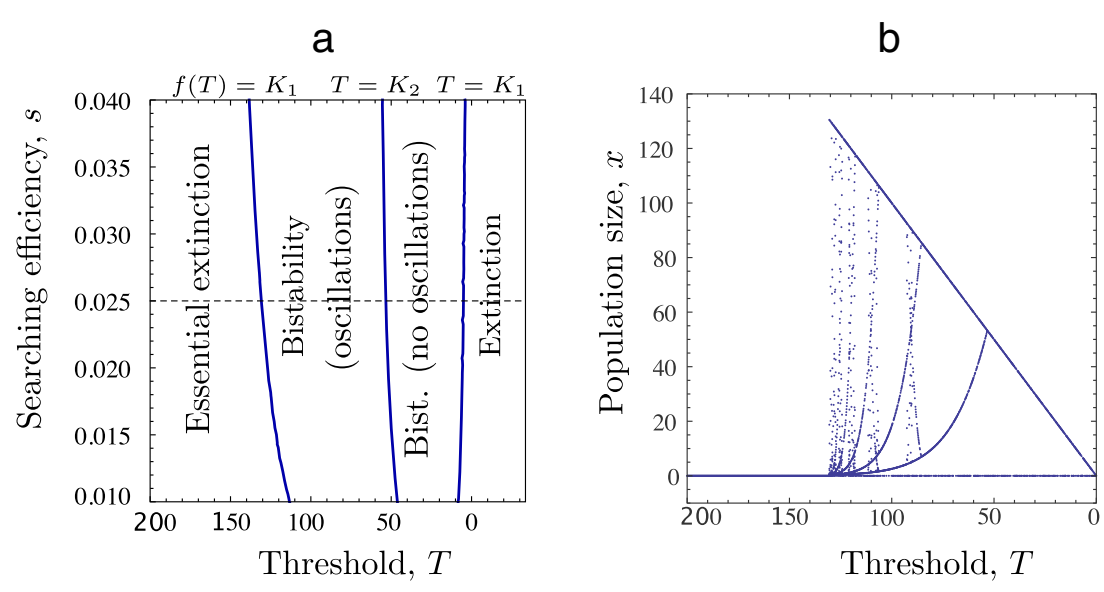
Fig. 6 Bifurcation diagrams (top row) and asymptotic yield (bottom row) for different harvesting strategies and the Ricker-Schreiber model (3.1) with $K=1, r=4, s=0.6$. For threshold harvesting, intervention starts at $T \approx 0.844$, the maximum value of $f$. In the top row, initial conditions are drawn from a pseudo-random uniform distribution. The bottom row is for an initial condition not leading to extinction
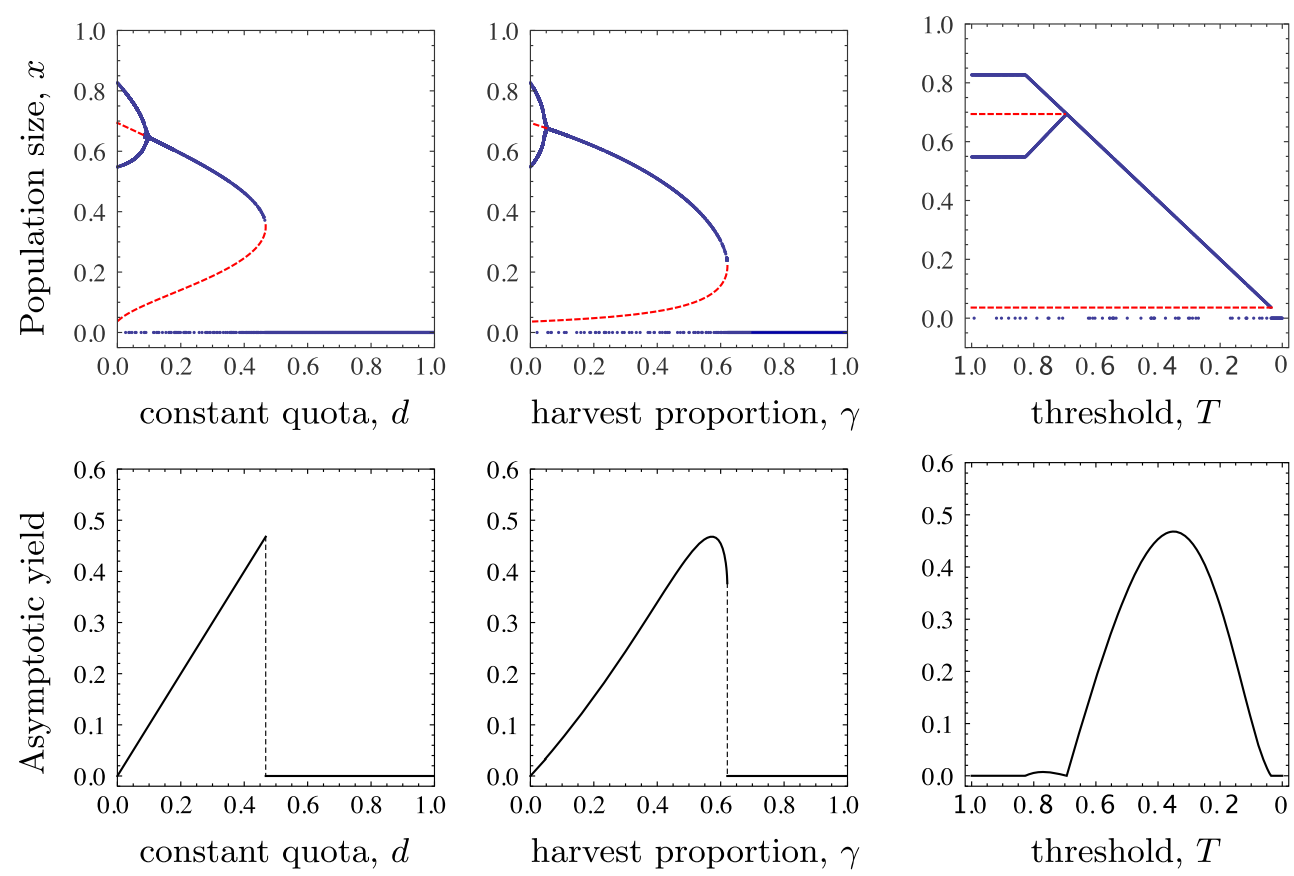

and Allee effects. Before a collapse occurs, there is an attracting equilibrium at which the population stabilizes its size for intermediate initial population values; higher values of this equilibrium make the collapse more dramatic, since the manager does not have early signals indicating the impending collapse. In our example, we can compute the population size at the bifurcation point leading to extinction.

1. For constant-quota harvesting, a collapse occurs if we harvest at a constant quota of $d=0.4678$. The equilibrium at the bifurcation point is $K_{2}(d) \approx 0.35$, which corresponds to $50 \%$ of the carrying capacity in the absence of harvesting $\left(K_{2} \approx 0.69\right)$.

2. For proportional harvesting, a collapse occurs if we harvest at a rate of $\gamma=62 \%$ of the population, and the equilibrium at the bifurcation point is $K_{2}(\gamma) \approx 0.22$, which corresponds to $31.8 \%$ of the carrying capacity in the absence of harvesting.

3. Finally, for threshold harvesting, a collapse occurs for $T=K_{1} \approx 0.036$, which is also the population size of the nontrivial attractor at the bifurcation point and corresponds to $5 \%$ of the carrying capacity in the absence of harvesting.

The Allee threshold corresponds to a tipping point of population sizes above which populations survive and below which populations go extinct. For constant-quota and proportional harvesting, the Allee threshold sharply increases with increasing harvesting intensity, especially near the bifurcation points (red dashed curves in Fig. 6). That is, the basin of attraction of the extinction state markedly expands due to these harvesting strategies. By contrast, the Allee threshold remains constant for increased threshold harvesting. As a consequence, under threshold harvesting, the population is more resilient against perturbations than that under the other harvesting strategies.

The asymptotic values of the average sustained yield is shown in Fig. 6. For constant-quota and proportional harvesting, the average yield collapses at or respectively shortly after the harvesting intensity producing maximum sustainable yield. For threshold harvesting, there is no abrupt collapse of the yield; it changes gradually even beyond the maximum sustainable yield and despite a strong Allee effect. This is a desirable feature for the adaptive management of developing fisheries (see Hilborn and Walters 1992). In the next section, we will investigate the shape of the yield curve for threshold harvesting in more detail.

\section{Average long-term yield}

An important feature of threshold harvesting (2.2) is that in many cases there is a periodic attractor which attracts all positive orbits with probability one. Moreover, the orbits become periodic after few transients. Thus, after these transients have died out, intervention takes place only once every $p$ years, where $p$ is the period of the attracting cycle. This property provides a simple method to calculate the frequency of harvest and the average long-term yield. 
In this Section, we consider again threshold harvesting (2.2) of a population with the generic condition (A), i.e., without Allee effect. Assume that

$\Gamma_{p}=\left\{T, f(T), \ldots, f^{p-1}(T)\right\}$

is the attracting cycle. Notice that $f^{i}(T)<T$ holds for all $i=1,2, \ldots, p-1$. Then, harvesting occurs only once every $p$ time steps, and what we remove from the population (harvest, yield) is

$f^{p}(T)-T$.

For example, if $T \leq K$, then $p=1$ such that there is an intervention every time step, and the average yield is $f(T)-T$.

If $f$ is differentiable, then it is easy to compute the threshold values that give the maximum long-term yield. Indeed, for a $p$-periodic window, we have to calculate the maximum of $g_{p}(x)=f^{p}(x)-x$, which is reached at the point $T$ such that $g_{p}^{\prime}(T)=0$, which is equivalent to $\mu=1$, where $\mu=f^{\prime}\left(q_{1}\right) f^{\prime}\left(q_{2}\right) \cdots f^{\prime}\left(q_{p}\right)$ is the multiplier associated to the cycle $\left\{q_{1}, q_{2}, \ldots, q_{p}\right\}$. For example, let us consider the Ricker map with $r=2.6$ and $K=60$ (Fig. 7). In the range $T \in(0,60), T$ is an attractor and the maximum harvest is attained at $T_{1} \approx 19.2$, with a yield value $h_{1} \approx$ 93.3. The 2-periodic window is $T \in(60,105.1)$, and the maximum yield within this threshold range is attained at $T_{2} \approx 84$, with a value of $h_{2} \approx 26.4$. However, since in this case harvesting occurs only once every two periods, the maximum average yield is $h_{2} / 2 \approx 13.2$.

That is, for threshold values $T \leq K$, the average longterm yield shows a dome-shaped form, which has been reported before for different types of population models

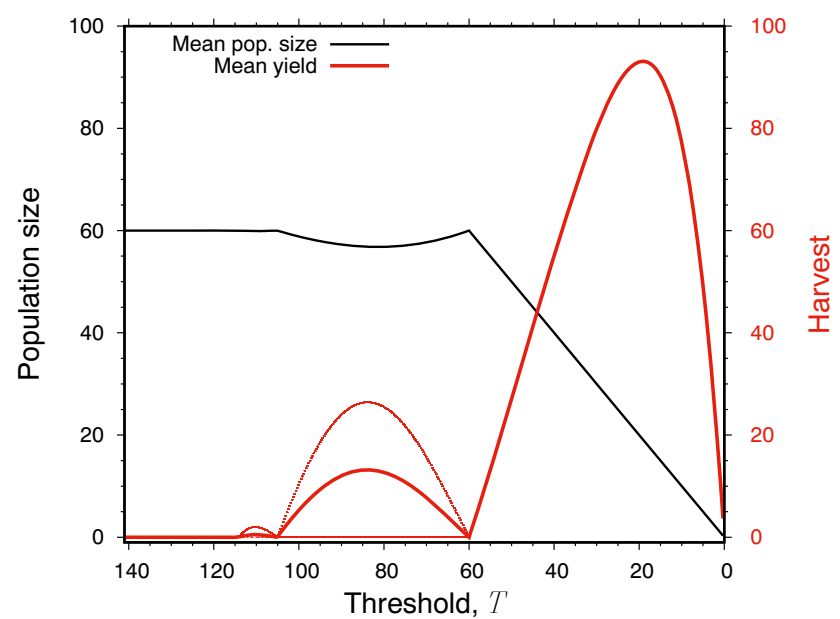

Fig. 7 Bifurcation diagram with the long-term harvest (thin red), average long-term yield (bold red), and mean population size (solid black). Threshold harvesting strategy (2.2) with the same Ricker map as in Fig. 3 (e.g., Hall et al. 1988; Lande et al. 1995). Here, we find the existence of additional dome-shaped relationships between average long-term yield and threshold in parameter ranges $T>K$ when the population dynamics is unstable in the absence of harvesting. To our knowledge, these additional local maxima in sustained yield have not been reported before for threshold harvesting (but see Hilker and Liz 2019 for the strategy of proportional threshold harvesting).

Figure 7 also shows the mean population size. We briefly report that there is a hydra effect (Abrams 2009; Hilker and Liz 2013) for $T \in(60,81.4)$, approximately. That is, the mean population size increases when harvesting mortality is enhanced within this parameter range.

\section{Stochastic simulations}

There is intrinsic uncertainty in population dynamics, assessment, and harvesting. This uncertainty is known to be problematic for harvesting and sustainable resource use (Hine and Gifford 1996; Roughgarden and Smith 1996; Gustafsson et al. 1999; Adamson and Hilker 2020). In this section, we consider three sources of random variation, namely demographic and environmental stochasticity (chance events and variation of external conditions) as well as observation error (generated by the measurement process).

\section{Model description}

Environmental stochasticity enters each generation in the form of a first-order autoregressive model:

$\varepsilon_{n+1}= \begin{cases}\rho \varepsilon_{n}+\sigma_{\mathrm{env}} \delta_{n+1} & \text { for } n>0 \\ \delta_{n+1} & \text { for } n=0\end{cases}$

where

$\delta_{n} \sim \operatorname{Normal}\left(\frac{-\sigma_{\mathrm{env}}^{2}}{2} \times \frac{1-\rho}{\sqrt{1-\rho^{2}}}, \sigma_{\mathrm{env}}^{2}\right)$

is an uncorrelated, normally distributed process error with a mean that corrects the bias from the autocorrelation in the environmental variation (Thorson et al. 2014). Parameter $\sigma_{\text {env }}>0$ determines the magnitude of environmental variation. $\rho \in(0,1)$ is the strength of the autocorrelation. If $\rho=0$, there is no autocorrelation and the noise is white. If $\rho$ is strictly positive (negative), the noise spectrum is reddened (blue-shifted). Here, we assume temporally autocorrelated environmental variations, as this has been considered important in ecosystems (Steele 1985) and for the choice of management strategies (Hall et al. 1988; Koslow 1989; Walters and Parma 1996; Kaitala et al. 2003). The individual reproduction is drawn from a Poisson distribution with a 
mean given by the reproduction curve $f\left(x_{n}\right)$, modulated by the log-normally distributed environmental noise. The population dynamics before harvesting is then described by

$\tilde{x}_{n}=\operatorname{Poisson}\left(f\left(x_{n}\right) e^{\varepsilon_{n}}\right)$,

which gives integer values as population abundance.

The population size in the next generation

$x_{n+1}=\tilde{x}_{n}-H_{n}$

is the one after harvesting, where the harvest in the current generation is given by

$H_{n}= \begin{cases}\tilde{x}_{\mathrm{obs}, n}-T & \text { if } \tilde{x}_{\mathrm{obs}, n}>T \\ 0 & \text { else }\end{cases}$

and based on the observed population size

$\tilde{x}_{\mathrm{obs}, n}=\tilde{x}_{n} \exp \left(\sigma_{\mathrm{obs}} \omega_{n}-\frac{\sigma_{\mathrm{obs}}^{2}}{2}\right)$,

where $\omega_{n}$ is drawn from a standard normal distribution. That is, the harvest is stochastic as it includes observation uncertainty (also called partial observability), which is assumed to be log-normally distributed (Hilborn and Mangel 1997).
We do not explicitly incorporate implementation error (also called partial controllability) but note that the stochastic harvest may be considered to subsume both observation and implementation error (cf. Jonzén et al. 2002). For proportional harvesting, $H_{n}=\gamma \tilde{x}_{\mathrm{obs}, n}$. For constant-quota harvesting, $H_{n}=d$ is assumed to be independent of population size estimates.

\section{Environmental stochasticity}

In the following Monte-Carlo simulations, we keep the autocorrelation in the environmental variation at a fixed value of $\rho=0.43$, which is the mean from a metaanalysis of 154 fish stocks (Thorson et al. 2014). For the moment, we ignore observation error but will investigate its impact separately later on. We have varied the strength of environmental stochasticity. Figure 8 shows results for three cases. The intermediate one is $\sigma_{\text {env }}=0.74$, which corresponds to the mean from the fish stock meta-analysis (Thorson et al. 2014). As fish populations tend to show substantial environmental variation in their recruitment, we also consider $\sigma_{\text {env }}=0.4$ as a lower value (which may still
Fig. 8 Comparison of threshold harvesting (left column) and proportional harvesting (right column) for different levels of environmental stochasticity (first row $\sigma_{\text {env }}=0.4$, second row $\sigma_{\text {env }}=0.74$, third row $\sigma_{\text {env }}=1$ ). Shown are the coefficient of variation of the harvest, the mean harvest scaled by the carrying capacity, the extinction risk, and the harvest frequency. Model Eqs. (5.1)-(5.6) with the Ricker map for $r=2.6$, $K=60, \rho=0.43$ including demographic stochasticity but no observation error $\left(\sigma_{\mathrm{obs}}=0\right)$. Results are based on 10,000 replicates and 100 iterations for each harvesting intensity, with the initial 50 iterations discarded to remove transient effects. Random initial conditions
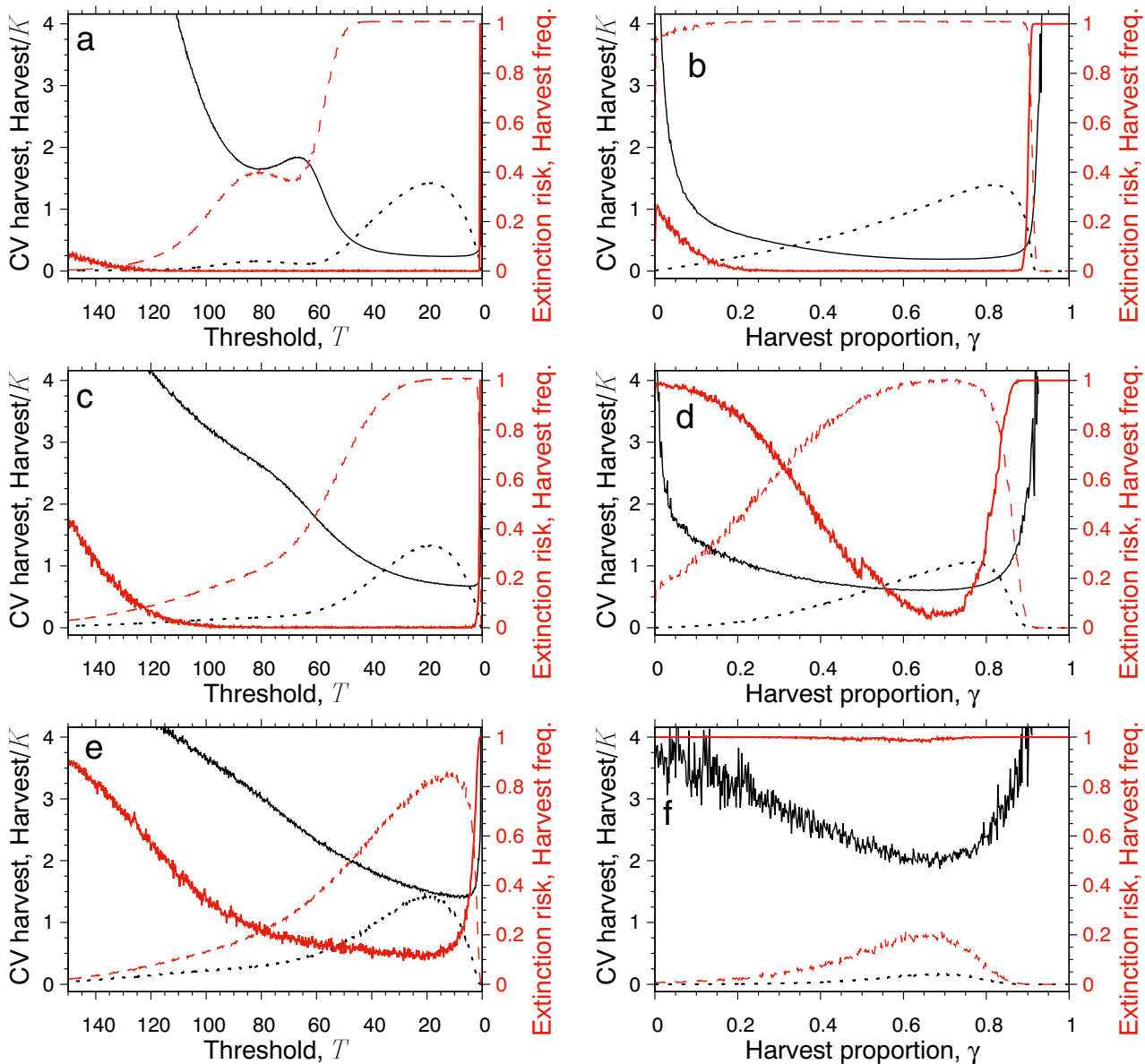

_ CV harvest $\quad \ldots$ Harvest $/ K$ 
be considered high for other taxa or certain species) and $\sigma_{\text {env }}=0.9$ as a higher value.

We would like to highlight the following observations in Fig. 8. First, consider extinction risk, which we define as the proportion of simulation replicates leading to extinction $\left(x_{t}<1\right)$ over the time horizon of 100 generations. Increased threshold harvesting, i.e., a lower escapement, decreases the extinction risk (solid red curves in Fig. 8a, c, e). This is because threshold harvesting curtails large population sizes that could trigger an overcompensatory population response and generate vulnerably small population sizes in the next generation. We note that the inverse of extinction risk is another measure of persistence stability, which suggests that smaller escapements enhance persistence stability. For excessively small escapement levels close to nil, however, the extinction risk rises sharply due to plain overexploitation.

Proportional harvesting implies an elevated extinction risk compared to threshold harvesting (see Fig. 8b, d, f). This is because proportional harvesting is applied at all population sizes, even if they are already small and will be further reduced by proportional harvesting. For large environmental stochasticity, almost all simulated populations have gone extinct under proportional harvesting (Fig. 8f). For small and medium environmental stochasticity, increased proportional harvesting can reduce extinction risk provided it is not too intense. This is because proportional harvesting stabilizes the population oscillations. However, if the harvesting proportion is too large, the extinction risk rises again due to overexploitation (Fig. 8b, d). This may occur well before maximum sustainable yield is achieved (Fig. 8d) and over a wider parameter range than for threshold harvesting. Hence, proportional harvesting seems inferior in promoting persistence stability compared to threshold harvesting. For constant-quota harvesting, basically all simulated populations have gone extinct, which is why the results for this harvesting strategy are not included in this Section.

Second, we observe that harvest frequency tends to increase with increased threshold harvesting, i.e., for lower escapements (see dashed red curves in Fig. 8a, c, e). For small environmental stochasticity, harvest frequency follows the deterministic staircase pattern predicted by our analytical results in "Average long-term yield," but with the sharp transitions smoothened out by the random variations (Fig. 8a). The latter can have twofold effects. On the one hand, they lead to population sizes below the threshold so that there is no harvesting. This is why there may be occasionally no harvesting for $T<K$, when harvesting would always occur in the deterministic system. However, harvest frequency is almost $100 \%$ when the threshold does not exceed about $80 \%$ of the carrying capacity. On the other hand, noise can lead to large population sizes so that there is harvesting even for large threshold values way in excess of the carrying capacity where there would be no harvesting in deterministic systems. For larger environmental stochasticity, the noise smears out the deterministic pattern even more, so that even low escapements do not guarantee harvest in each generation (Fig. 8f).

Proportional harvesting occurs in each generation, provided the population has not gone extinct. The earlier a population goes extinct, the lower the harvest frequency. This is why proportional harvesting does not always have a $100 \%$ harvest frequency in the stochastic models (Fig. 8b, $\mathrm{d}, \mathrm{f})$. Note that the frequency of proportional harvesting is overall higher than that of threshold harvesting for small environmental stochasticity (compare Fig. 8a and b), but that it is generally lower than that of threshold harvesting for large environmental stochasticity (compare Fig. 8e and $\mathrm{f}$ ). This runs counter to the usual notion that threshold harvesting implies more harvest closures. The reason is that threshold harvesting can sustain populations that would go extinct under proportional harvesting.

Third, increased threshold harvesting can reduce the variability in yield, measured by its coefficient of variation (solid black curves in Fig. 8). In other words, the predictability of the catch increases when setting lower escapements; this holds for a wide range of escapement values with an exception near the carrying capacity for low environmental stochasticity (Fig. 8a), as the deterministic population would never be harvested at this equilibrium. For larger environmental stochasticity, the harvest variability decreases throughout with lower escapements (Fig. 8c, e). However, the relative variation in yield increases in the unlikely case that the escapement approaches nil so that the population would be harvested deterministically to extinction.

For proportional harvesting, yield variability increases with harvesting intensity when the latter is high. For low and medium harvesting intensities, increased proportional harvesting reduces yield variability. This is in contrast with the classical result that yield variability increases with increased harvesting intensity for logistically growing populations in random, uncorrelated environments (Beddington and May 1977). The reason for this difference may be that increased proportional harvesting stabilizes the endogenous population oscillations generated by the overcompensatory population dynamics: the less variable the population size, the less variable the yield.

Note that for both threshold and proportional harvesting stronger environmental stochasticity enhances yield variability. While the yield variability under threshold harvesting is larger than under proportional harvesting for lower environmental stochasticity (compare Fig. 8a and b), it is the other way around for higher environmental stochasticity (compare Fig. 8e and f). This contradicts the usual notion that threshold harvesting comes with higher yield variability 
than proportional harvesting in the absence of observation error (Deroba and Bence 2008).

Fourth, threshold harvesting maximizes the mean harvest at escapement values that are indistinguishable for the considered levels of environmental stochasticity (see dotted black curves in Fig. 8a, c, e). Previous research on population models with continuous-time logistic growth predicts that the escapement value maximizing the mean harvest increases with enhanced environmental stochasticity (Lande et al. 1997). For continuous-time population models, in which density-dependence begins to act only close to the carrying capacity, the escapement value maximizing mean harvest decreases with increasing environmental stochasticity (Sæther et al. 1996). Hence, these results may not be applicable to the discrete-time overcompensatory population models considered here.

With proportional harvesting, the harvesting intensities maximizing mean harvest decrease with stronger environmental stochasticity (Fig. 8b, d, f). This agrees with theory based on continuous-time logistic population growth (Beddington and May 1977). Note that the maximum mean harvest decreases considerably with increasing environmental stochasticity for proportional harvesting, but remains relatively unaffected for threshold harvesting (Fig. 8).

\section{Observation error}

Estimates of population size are usually inaccurate. Coefficients of variation due to sampling vary from $10 \%$ to almost $60 \%$ for some species of fish, birds, and mammals (see
Table 6.2 in Lande et al. 2003). In their review of harvesting strategies, Deroba and Bence (2008) find that observation error has a particularly strong effect on the performance of harvesting strategies. The presence of observation error has been shown to change the relative performance of alternative harvesting strategies (see Table 1 in Deroba and Bence 2008), indicating that proportional harvesting can outperform threshold harvesting in terms of higher yields when there are observation error and autocorrelated environmental stochasticity. In the remaining part of this Section, we fix the levels of environmental stochasticity and autocorrelation at the mean values from the meta-analysis by Thorson et al. (2014), i.e., $\sigma_{\text {env }}=0.74$ and $\rho=0.43$. We will vary the strength of observation uncertainty measured in terms of $\sigma_{\text {obs }}$.

Figure $9 \mathrm{a}$ and $\mathrm{b}$ assume a $30.7 \%$ coefficient of variation due to observation error $\left(\sigma_{\mathrm{obs}}=0.3\right)$. This level of observation uncertainty causes elevated extinction risk and increased harvest variability for both threshold and proportional harvesting compared to the case with no observation error (compare Fig. 9a with Fig. 8c and Fig. 9b with Fig. 8d, respectively). The mean harvest and harvest frequency are both reduced with stronger observation uncertainty under proportional harvesting (Fig. 9b) but remain essentially unchanged under threshold harvesting (Fig. 9a). These results are in line with some reports in the literature that observation error seems to favor threshold harvesting over proportional harvesting in uncorrelated environments (Eggers 1993; Sladek Nowlis and Bollermann 2002) but contradict other studies (e.g., Butterworth and Bergh 1993; Walters and Parma 1996; Polacheck et al. 1999).
Fig. 9 Comparison of threshold harvesting (left column) and proportional harvesting (right column) for different levels of observation uncertainty (top row $\sigma_{\text {obs }}=0.3$, bottom row $\left.\sigma_{\mathrm{obs}}=0.55\right)$. Shown are the coefficient of variation of the harvest, the mean harvest scaled by the carrying capacity, the extinction risk, and the harvest frequency. Model

Eqs. (5.1)-(5.6) with the Ricker map for $r=2.6, K=60$, $\rho=0.43$ including demographic and environmental stochasticity $\left(\sigma_{\text {env }}=0.74\right)$. Results are based on 10,000 replicates and 100 iterations for each harvesting intensity, with the initial 50 iterations discarded to remove transient effects. Random initial conditions
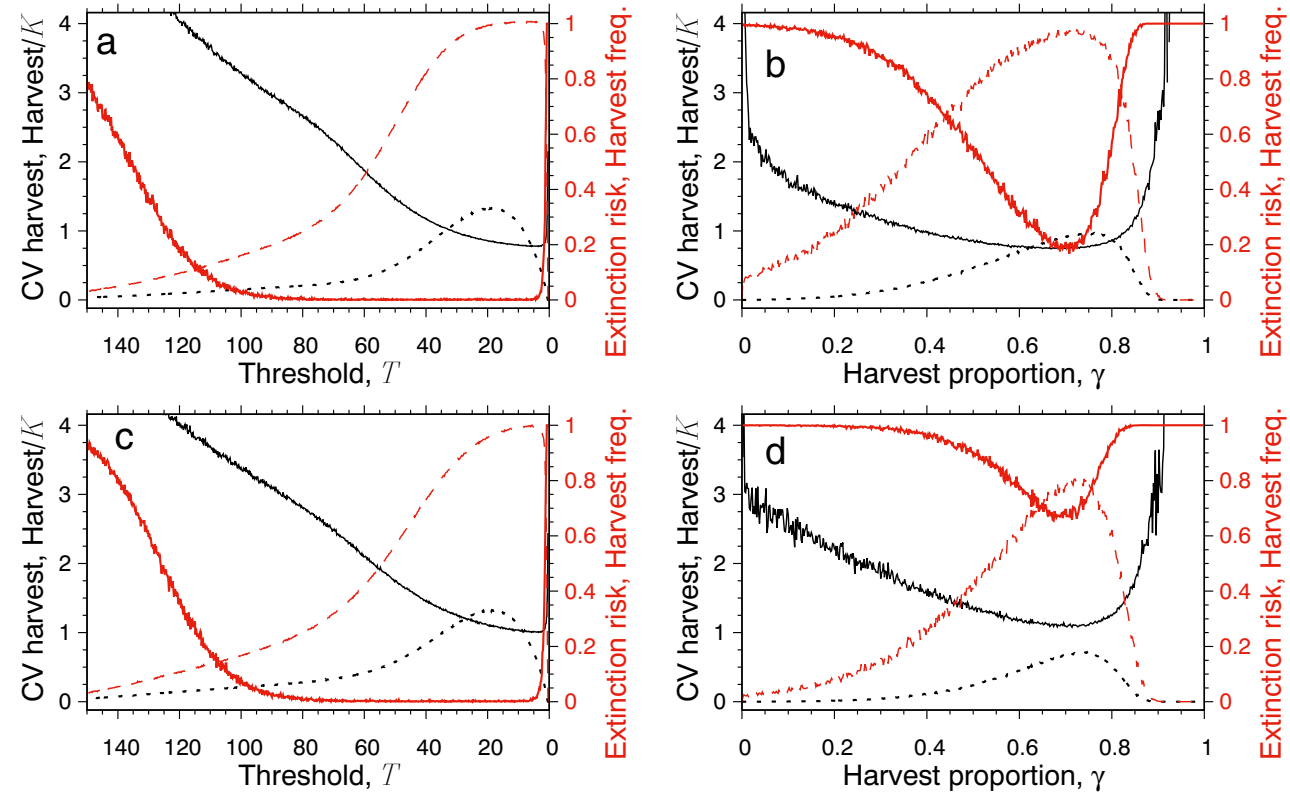

_ CV harvest $\quad \ldots$ Harvest $/ K$

Harvest freq. 
Further increasing the observation error to a $59.4 \%$ coefficient of variation $\left(\sigma_{\text {obs }}=0.55\right)$ affects the performance of threshold harvesting marginally (compare Fig. 9c with a). By contrast, the increased observation uncertainty causes almost always extinction for proportional harvesting (Fig. 9d). This suggests that threshold harvesting may be more robust against observation uncertainty than proportional harvesting.

\section{Conclusions}

As it is now widely recognized that harvesting and intrinsic population dynamics as well as environmental factors jointly impact the size and variability of populations (e.g.,
Bjørnstad and Grenfell 2001; Fromentin et al. 2001; Shelton and Mangel 2011), we have considered a range of ecological models and environmental conditions including iteroparity, strong Allee effects, and demographic and environmental stochasticity as well as observation uncertainty. Table 2 summarizes the main results of this paper. The emerging theme is that threshold harvesting tends to enhance multiple stability dimensions (dynamic, constancy, and persistence stability) and seems more resilient and robust against abrupt collapses than proportional and constant-quota harvesting. Our analytical results in this respect are quite general and apply to deterministic discrete-time single-species models. Our simulation results focus on overcompensatory population dynamics that are oscillatory. Overcompensation has been observed in insects (Nicholson 1957; Costantino

Table 2 Summary of main results

Populations without Allee effect

(analytical results for semelparous species satisfying condition (A))

- TH is never destabilizing ("Dynamic stability")—-this also holds for iteroparous species ("Bimodal maps with iteroparity").

- TH guarantees a globally asymptotically stable equilibrium for $T \leq K$ (Prop. 2.1) and the existence of period halvings when the carrying capacity is unstable for $T>K$; the latter result additionally requires $f$ to be an SU map ("Dynamic stability").

- Constancy stability (measured by fluctuation range) always increases when $T$ is lowered; this does not apply to iteroparous species with cycles of a period > 2 ("Constancy and persistence stability").

- Persistence stability (measured by inverse of lowest population abundance) increases for $T>K$ and decreases for $T<K$

("Constancy and persistence stability").

Populations with Allee effect

(simulation results for the Ricker-Schreiber map)

- TH is able to prevent essential extinction (Fig. 5); the required threshold value is easy to find analytically ("Models with Allee effect").

- If a sudden collapse occurs under TH, this is less dramatic than for PH and $\mathrm{CH}$ (Fig. 6).

- Allee threshold is only marginally increased by TH in comparison to PH and CH (Fig. 6).

- Dome-shaped yield curve without abrupt collapse amenable for adaptive management (Fig. 6).

Yield

(semelparous species satisfying condition (A))

- Analytical formulas of average long-term yield, including MSY, and long-term harvest frequency as a function of threshold values (for periodic systems; "Average long-term yield")

- There are additional local maxima in sustained average yield for $T>K$ (“Average long-term yield").

Random fluctuations

(simulation results for model (5.1)-(5.6) with the Ricker map, $r=2.6, K=60, \rho=0.43$ )

- Lower escapements tend to enhance persistence stability (by reducing extinction risk), reduce harvest closures (i.e., increase harvest frequency), and increase yield predictability (by reducing harvest variability) (Figs 8 and 9).

- The escapement level producing maximum sustainable yield comes with low extinction risk, almost no harvest closures, and is robust against levels of environmental variation and observation uncertainty (Figs. 8 and 9).

- TH sustains the population in many cases where PH and CH lead to population collapse (Figs. 8 and 9). 
et al. 1995), fish (Zipkin et al. 2008; Foss-Grant et al. 2016), plants (Thrall et al. 1989; Buckley et al. 2001; Pardini et al. 2009), and ungulates (Grenfell et al. 1992).

When overcompensation produces small population sizes, there is an increased risk of extinction, especially in stochastic environments. In our stochastic simulations (limited to certain life-history and autocorrelation parameters), threshold harvesting emerges as a more sustainable strategy than proportional and constant-quota harvesting. More specifically, threshold harvesting tends to sustain the population in conditions where proportional and constantquota harvesting lead to population extinction. The underlying reason is that threshold harvesting is stopped below the escapement and does not further reduce small population sizes. Proportional and constant-quota harvesting lack a built-in protection of small population sizes that may occur as a result of overcompensation, random variation, or observation uncertainty.

If threshold harvesting is used as a strategy for reducing intrinsic population fluctuations, then a threshold value near the carrying capacity seems a good choice as this is predicted to maintain a mean population size similar to the unharvested population and to have little burden in terms of having to harvest. This has been observed in deterministic (see Fig. 7; harvest frequency not explicitly shown here) as well as stochastic models (see Figs. 8, 9; mean population size not explicitly shown here) and also in ciliate experiments (Fryxell et al. 2005). An escapement level set at the carrying capacity may also be attractive for controlling pest species that erupt due to unstable dynamics. In a comparison of six control strategies to reduce fluctuations, simulations by Tung et al. (2014) showed that threshold harvesting with a threshold larger than the carrying capacity leads to the lowest population sizes when compared to the other control strategies, while removing the largest harvest for highly chaotic populations.

If management aims at reducing a pest population size as much as possible, or if the pest dynamics is stable, then thresholds far below the carrying capacity are advisable, but they come at the cost of almost always having to harvest, in both deterministic and stochastic models. The fruit fly experiments by Tung et al. (2016) implemented such low threshold values, but they found almost no effect on the average population size. However, their population census was taken just before harvesting and is therefore affected by increased population growth after reduced competition due to harvesting (a "hidden" hydra effect, see Hilker and Liz 2013).

If threshold harvesting is used as an exploitation strategy, the maximum sustainable yield is achieved at thresholds far below the carrying capacity. On the experimental side, neither the ciliate (Fryxell et al. 2005) nor the fruit fly microcosms (Tung et al. 2016) showed evidence for the dome-shaped relationship between yield and harvesting effort (here: lower thresholds) that is ubiquitously found in deterministic and stochastic models. This may be due to the limited number of harvesting efforts that were investigated. Tung et al. (2016) used two threshold values, which are definitely too few to find a dome-shaped relationship. Fryxell et al. (2005) found a monotonic rather than a domeshaped relationship, but the four threshold values that they used (corresponding to $100 \%, 75 \%, 50 \%$, and $25 \%$ of the carrying capacity) may have also been chosen at levels not revealing the full relationship.

In the literature, threshold harvesting is known to not only produce higher yields than other harvesting strategies, but also maintain larger and less variable population sizes in many circumstances (Ricker 1958; Gatto and Rinaldi 1976; Getz and Haight 1989; Lande et al. 1997; Deroba and Bence 2008). However, this comes at the cost of increased yield variability, which is attributed to fishery closures or ceased harvesting when the population size falls below the threshold (Lande et al. 1997; Lillegård et al. 2005). The simulations in this paper suggest that harvesting closures are unlikely around thresholds producing MSY, as harvest frequency approaches 100\% for thresholds of about $80 \%$ the carrying capacity or lower. The threshold level producing MSY and the MSY level itself are remarkably invariant to environmental variation and observation error in the simulations performed. Here, threshold harvesting outperforms both proportional and constant-quota harvesting with respect to average yield (and also yield constancy) in the presence of large environmental stochasticity and observation error.

Such threshold values are well within the range recommended in the fisheries literature (see Deroba and Bence 2008, Sect. 6.4). However, in this range of threshold values, the population variability basically vanishes, which makes stock assessment more difficult (Hilborn and Walters 1992). The strategy of proportional threshold harvesting adopts many features of threshold harvesting, but allows the population size to vary over a wider range (Engen et al. 1997; Hilker and Liz 2019).

Moreover, there is the need and difficulty of knowing the population size for applying threshold harvesting. Measurement error and uncertainty have been shown to change the optimal strategy for economic exploitation (e.g., Clark and Kirkwood 1986; Sethi et al. 2005; Deroba and Bence 2008) and to exacerbate the biological problem of overfishing (Roughgarden and Smith 1996). Haydon and Fryxell (2004) show that reduced uncertainty by increased understanding and prediction of population variation will have only a modest effect on absolute yield, but may substantially reduce the risk of overexploitation.

All these results suggest that the choice of threshold values or management strategy in the first place depends on 
a suite of socio-economic, ecological, and political factors. Many comparisons of harvesting strategies are based on certain objectives such as maximizing yield, sometimes also taking into account other factors (cf. Milner-Gulland 1998; Deroba and Bence 2008). Here, we have used dynamical system approaches and numerical bifurcation analyses to investigate population and harvest stability over an entire range of threshold values. This is likely to give a more holistic picture of system behavior than simulations based on an optimization criterion only and complements experiments performed with a limited set of threshold values. However, all of our results are based on single-species models. They ignore age structure, which is known to affect stability results already in simple harvesting models (e.g., Zipkin et al. 2009; Liz and Pilarczyk 2012). And they account neither for simultaneous continuoustime processes nor for ecosystem interactions. There is increasing awareness to develop harvest control rules within the context of ecological communities (e.g., Quinn and Collie 2005; Walters et al. 2005; Matsuda and Abrams 2006). Travis et al. (2014) argue that the neglect of species interactions in fisheries science and management may be responsible for population collapse and cascading effects in ecosystems.

Funding information Open Access funding provided by Projekt DEAL. Eduardo Liz acknowledges the support of the research grant MTM2017-85054-C2-1-P (AEI/FEDER, UE).

\section{Appendix A}

\section{A.1 Proof of Proposition 2.1}

The following proves Proposition 2.1 in the main text. The assumptions made in this proposition are that $T \leq K$ and condition (A) holds, that is, $f$ is continuous and has a unique fixed point $K$, with $f(x)>x$ for all $x \in(0, K)$ and $f(x)<x$ for all $x>K$.
Since $g(x)=\min \{f(x), T\}$, it is clear that $g(x)>x$ for all $x \in(0, T)$, and $g(x) \leq T<x$ for all $x>T$. Thus, $T$ is the unique fixed point of $g$ in $(0, \infty)$. Choose an initial condition $x_{0}>0$. It is evident that $g^{n}\left(x_{0}\right) \leq T<K$, for all integer $n \geq 1$. Since $g(x) \geq x$ for all $x \leq T$, it follows that the sequence $\left\{g^{n}\left(x_{0}\right)\right\}_{n \geq 1}$ is nondecreasing and bounded, and therefore converges to a limit. By continuity, the limit is a positive fixed point of $g$ and hence $\lim _{n \rightarrow \infty} g^{n}\left(x_{0}\right)=T$.

\section{A.2 Period-halving bifurcations for simple unimodal maps}

We recall that the Schwarzian derivative of a $\mathcal{C}^{3}$ map $f$ is defined by the expression

$(S f)(x)=\left(\frac{f^{\prime \prime \prime}(x)}{f^{\prime}(x)}\right)-\frac{3}{2}\left(\frac{f^{\prime \prime}(x)}{f^{\prime}(x)}\right)^{2}$,

whenever $f^{\prime}(x) \neq 0$.

Many maps usually employed in discrete-time models of population dynamics are $S U$-maps. This means that they have a unique critical point $c$ (which corresponds to a local maximum) and $(S f)(x)<0$ for all $x \neq c$. Well-known examples of SU-maps are the Ricker map $f(x)=x e^{r(1-x)}$, $r>0$, the quadratic map $f(x)=r x(1-x), r>1$, and the Bellows map $f(x)=a x /\left(1+x^{m}\right)$ for $a>1$ and $m \geq 2$.

The fact that $f$ is an SU-map simplifies the dynamics in various ways; perhaps the most relevant fact is that it ensures that $f$ and its iterates $f^{n}, n \geq 2$, have at most one inflection point in each interval without critical points, and this is a key property to limit the number of attractors. For example, an asymptotically stable fixed point $K$ of a map $f$ satisfying (A) is a global attractor if $f^{2}$ has no other fixed point than $K$. Additional fixed points of $f^{2}$ can be easily excluded for SU-maps if $K$ is asymptotically stable, but they can appear for general unimodal maps. For example, the Ricker map $f(x)=x e^{1.885(1-x)}$ has a positive
Fig. 10 Plots of the graph $y=f^{2}(x)=f(f(x))$ of the second iteration of $f$ (blue curve), and the line $y=x$ (black). A: $f(x)=x e^{1.885(1-x)}$ is an SU-map. B: $f(x)=7.86 x-23.31 x^{2}+$ $28.75 x^{3}-13.30 x^{4}$ is unimodal but not an SU-map a

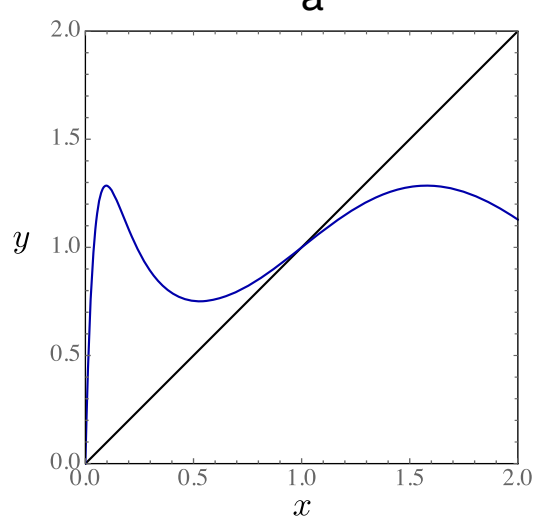

b

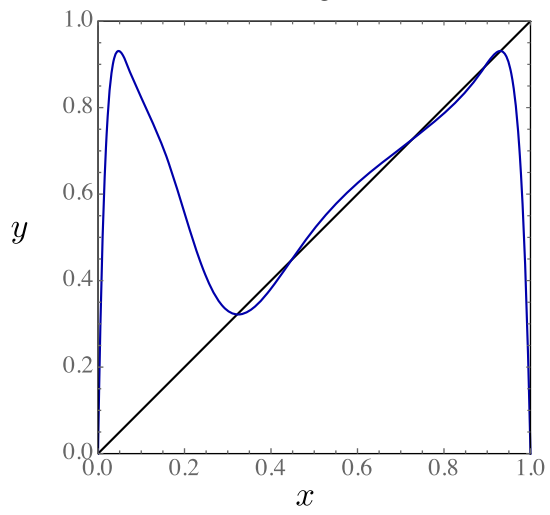


equilibrium $K=1$ with $f^{\prime}(K)=-0.885$, and hence, it is asymptotically stable. Since $f$ is an SU-map, $K$ is the only fixed point of $f^{2}$, and therefore, it is a global attractor of $f$ (Fig. 10a). However, the unimodal map $f:[0,1] \rightarrow[0,1]$ defined by $f(x)=7.86 x-23.31 x^{2}+28.75 x^{3}-13.30 x^{4}$ has a unique positive equilibrium $K \approx 0.726$, with $f^{\prime}(K) \approx$ -0.885 , as in the previous example. But $f$ is not an SUmap and $f^{2}$ has five positive fixed points (Fig. 10b). Of course, $K$ is not a global attractor for $f$ in this case, since an attracting 2-cycle coexists with the attracting fixed point. This polynomial has been used in Singer (1978) to illustrate the importance of the Schwarzian derivative.

A more general result establishes that SU-maps satisfying condition (A) have at most one attracting cycle (see Theorem 5.1 and Corollary 5.9 in Sharkovsky et al. 1997). Assuming that the map $f$ has only a finite number of periodic orbits, Theorem 5.3 in the same reference ensures that the attracting cycle has period $2^{N}$, for an integer $N \geq 0$, and $f$ has only cycles of period $2^{i}$, with $0 \leq i \leq N$. Furthermore, the properties of simple cycles (Sharkovsky et al. 1997, Chapters 3 and 4) guarantee the simple structure of the period-halving bifurcations that occur as $T$ is decreased: an attracting cycle $\left\{x_{1}, x_{2}, \ldots, x_{p}\right\}\left(x_{1}<x_{2}<\cdots<x_{p}\right)$ of period $p=2^{i}$ disappears through a period-halving bifurcation when $T$ reaches the unique value $x_{q} \in\left(x_{p-1}, x_{p}\right)$ such that $f^{2^{i-1}}\left(x_{q}\right)=x_{q}$.

We give a flavor of these results, without going deeply into technical details.

A cycle of $f$ is defined by a permutation. For example, a 2-cycle $\left\{x_{1}, x_{2}\right\}$ of (minimal) period 2 is defined by the permutation of length 2

$\pi_{2}=\left(\begin{array}{ll}1 & 2 \\ 2 & 1\end{array}\right)$

which means that $f\left(x_{1}\right)=x_{2}$ and $f\left(x_{2}\right)=x_{1}$. The permutation $\pi_{2}$ is called minimal. The concept of minimal permutation can be defined by induction when the length of the permutation is an even number: a permutation of length 4 is minimal if the sets $\{1,2\}$ and $\{3,4\}$ are invariant under $\pi^{2}$ and the restriction of $\pi^{2}$ to each of these sets is minimal. If the length of $\pi$ is $p=2 k$, then the permutation $\pi$ is minimal if the sets $\{1,2, \ldots, k\}$ and $\{k+1, k+$ $2, \ldots, 2 k\}$ are invariant under $\pi^{2}$ and the restriction of $\pi^{2}$ to each of these sets is minimal. Examples of minimal permutations are

$\pi_{4}=\left(\begin{array}{llll}1 & 2 & 3 & 4 \\ 3 & 4 & 2 & 1\end{array}\right), \quad \pi_{8}=\left(\begin{array}{llllllll}1 & 2 & 3 & 4 & 5 & 6 & 7 & 8 \\ 5 & 6 & 7 & 8 & 3 & 4 & 2 & 1\end{array}\right)$.

Notice that

$\pi_{8}^{2}=\left(\begin{array}{llll|llll}1 & 2 & 3 & 4 & 5 & 6 & 7 & 8 \\ 3 & 4 & 2 & 1 & 7 & 8 & 6 & 5\end{array}\right)$ is composed of two minimal permutations of length 4 , and

$\pi_{8}^{4}=\left(\begin{array}{ll|ll|ll|ll}1 & 2 & 3 & 4 & 5 & 6 & 7 & 8 \\ 2 & 1 & 4 & 3 & 6 & 5 & 8 & 7\end{array}\right)$

is composed of four minimal permutations of length 2 .

A cycle of $f$ is of minimal type if its associated permutation is minimal. By Theorem 3.6 in Sharkovsky et al. (1997), if a map has only cycles whose periods are a power of 2, then all cycles are of minimal type. These maps are called simple maps, and their cycles are referred to as simple cycles.

The properties of simple cycles are responsible for the fine bifurcation structure of Eq. (2.2). For example, if $\left\{x_{1}, x_{2}, \ldots, x_{8}\right\}$ is the 8 -cycle associated to $\pi_{8}$, then $f^{4}\left(x_{7}\right)=x_{8}$ and $f^{4}\left(x_{8}\right)=x_{7}$. Thus, there exists $y_{4} \in$ $\left(x_{7}, x_{8}\right)$ such that $f^{4}\left(y_{4}\right)=y_{4}$. Moreover, $y_{4}=\max \{x>$ $\left.0: f^{4}(x) \geq x\right\}$.

Since the 4-cycle $\left\{y_{1}, y_{2}, y_{3}, y_{4}\right\}$ is simple, it follows that $f^{2}\left(y_{3}\right)=y_{4}$ and $f^{2}\left(y_{4}\right)=y_{3}$. Therefore, there exists $z_{2} \in\left(y_{3}, y_{4}\right)$ such that $f^{2}\left(z_{2}\right)=z_{2}$. Moreover, $z_{2}=$ $\max \left\{x>0: f^{2}(x) \geq x\right\}$. Finally, there exists a fixed point $K \in\left(z_{1}, z_{2}\right)$, where $z_{1}=f\left(z_{2}\right)$, and $K=\max \{x>0$ : $f(x) \geq x\}$.

The values $T_{1}=y_{4}, T_{2}=z_{2}$, and $T_{3}=K$ provide the period-halving bifurcation points of Eq. (2.2) as $T$ decreases.

Open Access This article is licensed under a Creative Commons Attribution 4.0 International License, which permits use, sharing, adaptation, distribution and reproduction in any medium or format, as long as you give appropriate credit to the original author(s) and the source, provide a link to the Creative Commons licence, and indicate if changes were made. The images or other third party material in this article are included in the article's Creative Commons licence, unless indicated otherwise in a credit line to the material. If material is not included in the article's Creative Commons licence and your intended use is not permitted by statutory regulation or exceeds the permitted use, you will need to obtain permission directly from the copyright holder. To view a copy of this licence, visit http:// creativecommonshorg/licenses/by/4.0/.

\section{References}

Abrams PA (2009) When does greater mortality increase population size? The long history and diverse mechanisms underlying the hydra effect. Ecol Lett 12:462-474

Adamson MW, Hilker FM (2020) Resource-harvester cycles caused by delayed knowledge of the harvested population state can be dampened by harvester forecasting. Theoretical Ecology, revised version submitted. https://doi.org/10.1007/s12080-020-00462-x

Allen KR (1973) The influence of random fluctuations in the stock recruitment relationship on the economic return from salmon fisheries. In: Parrish BB (ed) Fish stocks and recruitment. Conseil International pour l'Exploration de la Mer, Charlottenlund, pp 350-359

Barraquand F, Louca S, Abbott KC, Cobbold CA, Cordoleani F, DeAngelis DL, Elderd BD, Fox JW, Greenwood P, Hilker FM, Murray DL, Stieha CR, Taylor RA, Vitense K, Wolkowicz GS, 
Tyson RC (2017) Moving forward in circles: challenges and opportunities in modelling population cycles. Ecol Lett 20:10741092

Beddington JR, May RM (1977) Harvesting natural populations in a randomly fluctuating environment. Science 197:463-465

Bjørnstad ON, Grenfell BT (2001) Noisy clockwork: time series analysis of population fluctuations in animals. Science 293:638643

Buckley YM, Hinz HL, Matthies D, Rees M (2001) Interactions between density-dependent processes, population dynamics and control of an invasive plant species, Tripleurospermum perforatum (scentless chamomile). Ecol Lett 4:551-558

Butterworth D, Bergh M (1993) The development of a management procedure for the South African anchovy resource. In: Smith S, Hunt J, Rivard D (eds) Evaluation risk and biological reference points for fisheries management. vol 120 of Canadian special publication of fisheries and aquatic sciences. National Research Council Canada, Ottawa, pp 83-399

Cid B, Hilker FM, Liz E (2014) Harvest timing and its population dynamic consequences in a discrete single-species model. Math Biosci 248:78-87

Clark CW (1976) Mathematical bioeconomics the optimal management of renewable resources. Wiley, New York

Clark CW, Kirkwood GP (1986) On uncertain renewable resource stocks: optimal harvest policies and the value of stock surveys. J Environ Econ Manag 13:235-244

Collie JS, Spencer PD (1993) Management strategies for fish populations subject to long-term environmental variability and depensatory predation. In: Proceedings of the international symposium on management strategies for exploited fish populations, vol AKSG-93-02. Alaska Sea Grant, Fairbanks, pp 629-650

Corron NJ, Pethel SD, Hopper BA (2000) Controlling chaos with simple limiters. Phys Rev Lett 84:3835-3838

Costantino RF, Cushing JM, Dennis B, Desharnais RA (1995) Experimentally induced transitions in the dynamic behaviour of insect populations. Nature 375:227-230

Deroba JJ, Bence JR (2008) A review of harvest policies: understanding relative performance of control rules. Fish Res 94:201-233

Eggers DM (1993) Robust harvest policies for Pacific salmon fisheries. In: Proceedings of the international symposium on management strategies for exploited fish populations, vol AKSG93-02. Alaska Sea Grant, Fairbanks, pp 85-106

Enberg K (2005) Benefits of threshold strategies and age-selective harvesting in a fluctuating fish stock of Norwegian spring spawning herring Clupea harengus. Marine Ecological Progress Series 298:277-286

Engen S, Lande R, Sæther B-E (1997) Harvesting strategies for fluctuating populations based upon uncertain population estimates. J Theor Biol 186:201-212

Evans GT (1981) The potential collapse of fish stocks in a developing fishery. N Am J Fish Manag 1:127-133

Foss-Grant AP, Zipkin EF, Thorson JT, Jensen OP, Fagan WF (2016) Hierarchical analysis of taxonomic variation in intraspecific competition across fish species. Ecology 97:1724-1734

Fromentin J-M, Myers RA, Bjørnstad ON, Stenseth NC, Gjøsæter J, Christie H (2001) Effects of density-dependent and stochastic processes on the regulation of cod populations. Ecology 82:567-579

Fryxell JM, Smith IM, Lynn DH (2005) Evaluation of alternate harvesting strategies using experimental microcosms. OIKOS 111:143-149

Gatto M, Rinaldi S (1976) Mean value and variability of fish catches in fluctuating environments. J Fish Res Board Can 33:189-193

Getz WM, Haight RG (1989) Population harvesting: demographic models of fish, forest and animal resources. Princeton University Press, Princeton NJ
Glass L, Zeng W (1994) Bifurcations in flat-topped maps and the control of cardiac chaos. International Journal of Bifurcation and Chaos 4:1061-1067

Grenfell BT, Price OF, Albon SD, Glutton-Brock TH (1992) Overcompensation and population cycles in an ungulate. Nature 355:823-826

Grimm V, Wissel C (1997) Babel, or the ecological stability discussions: an inventory and analysis of terminology and a guide for avoiding confusion. Oecologia 109:323-334

Gustafsson M, Biel A, Gärling T (1999) Overharvesting of resources of unknown size. Acta Psychol 103:47-64

Hall DL, Hilborn R, Stocker M, Walters CJ (1988) Alternative harvest strategies for Pacific herring (Clupea harengus pallasi). Can J Fish Aquat Sci 45:888-897

Haydon DT, Fryxell J (2004) Using knowledge of recruitment to manage harvesting. Ecology 85:78-85

Hilborn R (1979) Comparison of fisheries control systems that utilize catch and effort data. J Fish Res Board Can 36:1477-1489

Hilborn R, Mangel M (1997) The ecological detective. Princeton University Press, Princeton

Hilborn R, Walters CJ (1992) Quantitative fisheries stock assessment: choice, dynamics and uncertainty. Chapman \& Hall, London

Hilker FM, Liz E (2013) Harvesting, census timing and "hidden" hydra effects. Ecol Complex 14:95-107

Hilker FM, Liz E (2019) Proportional threshold harvesting in discretetime population models. J Math Biol 79:1927-1951

Hilker FM, Westerhoff FH (2005) Control of chaotic population dynamics: ecological and economic considerations. Beiträge des Instituts für Umweltsystemforschung 32:1-21

Hilker FM, Westerhoff FH (2006) Paradox of simple limiter control. Phys Rev E 73:052901

Hine DW, Gifford R (1996) Individual restraint and group efficiency in commons dilemmas: the effects of two types of environmental uncertainty. J Appl Soc Psychol 26:993-1009

Jonzén N, Ripa J, Lundberg P (2002) A theory of stochastic harvesting in stochastic environments. Am Nat 159:427-437

Kaitala V, Jonzén N, Enberg K (2003) Harvesting strategies in a fish stock dominated by low-frequency variability: the Norwegian spring-spawning herring (Clupea harengus). Mar Resour Econ 18:263-274

Koslow JA (1989) Managing nonrandomly varying fisheries. Can J Fish Aquat Sci 46:1302-1308

Lande R, Engen S, Sæther B-E (1995) Optimal harvesting of fluctuating populations with a risk of extinction. Am Nat 145:728-745

Lande R, Engen S, Sæther B-E (2003) Stochastic population dynamics in ecology and conservation. Oxford University Press, Oxford

Lande R, Sæther B-E, Engen S (1997) Threshold harvesting for sustainability of fluctuating resources. Ecology 78:1341-1350

Larkin PA, Ricker WE (1964) Further information on sustained yields from fluctuating environments. J Fish Res Board Can 21:1-7

Lillegård M, Engen S, Sæther B-E, Toresen R (2005) Harvesting strategies for Norwegian spring-spawning herring. Oikos 110:567-577

Liz E (2010) Complex dynamics of survival and extinction in simple population models with harvesting. Theoretical Ecology 3:209221

Liz E, Pilarczyk P (2012) Global dynamics in a stage-structured discrete-time population model with harvesting. J Theor Biol 297:148-165

Liz E, Ruiz-Herrera A (2012) The hydra effect, bubbles, and chaos in a simple discrete population model with constant effort harvesting. J Math Biol 65:997-1016

Ludwig D (1979) Optimal harvesting of a randomly fluctuating resource. I: application of perturbation methods. SIAM J Appl Math 37:166-184 
Ludwig D (1980) Harvesting strategies for a randomly fluctuating population. Journal du Conseil pour l'Exploration de la Mer 39:168-174

Ludwig D (1998) Management of stocks that may collapse. Oikos $83: 397-402$

Ludwig D, Hilborn R, Walters C (1993) Uncertainty, resource exploitation, and conservation: lessons from history. Science 260(17):36

Mangel M (1985) Decision and control in uncertain resource systems. Academic Press, Orlando FL

Matsuda H, Abrams PA (2006) Maximal yields from multispecies fisheries systems: rules for systems with multiple trophic levels. Ecol Appl 16:225-237

Mendelssohn R (1979) Determining the best trade-off between expected economic return and the risk of undesirable events when managing a randomly varying population. J Fish Res Board Can 36:939-947

Milner-Gulland EJ, Mace R (1998) Conservation of biological resources. Wiley-Blackwell, Malden

Milner-Gulland EJ, Shea K, Possingham H, Coulson T, Wilcox C (2001) Competing harvesting strategies in a simulated population under uncertainty. Anim Conserv 45:157-167

Nicholson AJ (1957) The self-adjustment of populations to change. Cold Spring Harb Symp Quant Biol 22:153-173

Pardini EA, Drake JM, Chase JM, Knight TM (2009) Complex population dynamics and control of the invasive biennial Alliaria petiolata (garlic mustard). Ecol Appl 19:387-397

Polacheck T, Klaer NL, Millar C, Preece AL (1999) An initial evaluation of management strategies for the southern bluefin tuna fishery. ICES J Mar Sci 56:811-826

Quinn TJ, Collie JS (2005) Sustainability in single-species population models. Philos Trans R Soc B Biol Sci 360:147-162

Reed WJ (1978) The steady state of a stochastic harvesting model. Math Biosci 41:273-307

Reed WJ (1979) Optimal escapement levels in stochastic and deterministic harvesting models. J Environ Econ Manag 6:350-363

Reed WJ (1980) Optimum age-specific harvesting in a nonlinear population model. Biometrics 36:579-593

Ricker WE (1952) Numerical relations between abundance of predators and survival of prey. The Canadian Fish Culturist 13:5-9

Ricker WE (1954) Stock and recruitment. J Fish Res Board Can 11:559-623

Ricker WE (1958) Maximum sustained yields from fluctuating environments and mixed stocks. J Fish Res Board Can 15:9911006

Roughgarden J, Smith F (1996) Why fisheries collapse and what to do about it. Proc Natl Acad Sci 93:5078-5083

Sabo JL (2005) Stochasticity, predator-prey dynamics, and trigger harvest of nonnative predators. Ecology 86:2329-2343

Sæther B-E, Engen S, Lande R (1996) Density-dependence and optimal harvesting of fluctuating populations. Oikos 76:40-46

Schreiber SJ (2001) Chaos and population disappearances in simple ecological models. J Math Biol 42:239-260

Schreiber SJ (2003) Allee effects, extinctions, and chaotic transients in simple population models. Theor Popul Biol 64:201-209

Sethi G, Costello C, Fisher A, Hanemann M, Karp L (2005) Fishery management under multiple uncertainty. J Environ Econ Manag 50:300-318

Sharkovsky AN, Kolyada SF, Sivak AG, Fedorenko VV (1997) Dynamics of one-dimensional maps. Springer, Dordrecht
Shelton AO, Mangel M (2011) Fluctuations of fish populations and the magnifying effects of fishing. Proc Natl Acad Sci 108:70757080

Singer D (1978) Stable orbits and bifurcation of maps of the interval. SIAM J Appl Math 35:260-267

Sinha S (1994) Unidirectional adaptive dynamics. Phys Rev E 49:4832-4842

Sinha S (2001) Using thresholding at varying intervals to obtain different temporal patterns. Phys Rev E 63:036212

Sinha S, Parthasarathy S (1996) Unusual dynamics of extinction in a simple ecological model. Proc Natl Acad Sci 93:1504-1508

Sladek Nowlis J, Bollermann B (2002) Methods for increasing the likelihood of restoring and maintaining productive fisheries. Bull Mar Sci 70:715-731

Steele JH (1985) A comparison of terrestrial and marine ecological systems. Nature 313:355-358

Stoop R, Wagner C (2003) Scaling properties of simple limiter control. Phys Rev Lett 90:154101

Tautz A, Larkin PA, Ricker WE (1969) Some effects of simulated long-term environmental fluctuations on maximum sustained yield. J Fish Res Board Can 26:2715-2726

Thieme HR (2003) Mathematics in population biology. Princeton University Press, Princeton NJ

Thorson JT, Jensen OP, Zipkin EF (2014) How variable is recruitment for exploited marine fishes? A hierarchical model for testing life history theory. Can J Fish Aquat Sci 71:973-983

Thrall PH, Pacala SW, Silander JA (1989) Oscillatory dynamics in populations of an annual weed species Abutilon theophrasti. J Ecol 77:1135-1149

Thunberg H (2001) Periodicity versus chaos in one-dimensional dynamics. SIAM Rev 43:3-30

Travis J, Coleman FC, Auster PJ, Cury PM, Estes JA, Orensanz J, Peterson CH, Power ME, Steneck RS, Wootton JT (2014) Integrating the invisible fabric of nature into fisheries management. Proc Natl Acad Sci 111:581-584

Tung S, Mishra A, Dey S (2014) A comparison of six methods for stabilizing population dynamics. J Theor Biol 356:163-173

Tung S, Mishra A, Dey S (2016) Stabilizing the dynamics of laboratory populations of Drosophila melanogaster through upper and lower limiter controls. Ecol Complex 25:18-25

Walters C, Parma AM (1996) Fixed exploitation rate strategies for coping with effects of climate change. Can J Fish Aquat Sci 53:148-158

Walters CJ, Christensen V, Martell SJ, Kitchell JF (2005) Possible ecosystem impacts of applying MSY policies from single-species assessment. ICES J Mar Sci 62:558-568

Yakubu A-A, Li N, Conrad JM, Zeeman M-L (2011) Constant proportion harvest policies: dynamic implications in the Pacific halibut and Atlantic cod fisheries. Math Biosci 232:66-77

Zhou CT (2006) Stabilizing long-period orbits via symbolic dynamics in simple limiter controllers. Chaos 16:013109

Zhou CT, Yu MY (2005) Comparison between constant feedback and limiter controllers. Phys Rev E 71:016204

Zipkin EF, Kraft CE, Cooch EG, Sullivan PJ (2009) When can efforts to control nuisance and invasive species backfire? Ecol Appl 19:1585-1595

Zipkin EF, Sullivan PJ, Cooch EG, Kraft CE, Shuter BJ, Weidel BC (2008) Overcompensatory response of a smallmouth bass (Micropterus dolomieu) population to harvest: release from competition? Can J Fish Aquat Sci 65:2279-2292 